\title{
COMPLETE ELECTRODE MODEL OF ELECTRICAL IMPEDANCE TOMOGRAPHY: APPROXIMATION PROPERTIES AND CHARACTERIZATION OF INCLUSIONS*
}

\author{
NUUTTI HYVÖNEN ${ }^{\dagger}$
}

\begin{abstract}
In electrical impedance tomography one tries to recover the spatial admittance distribution inside a body from boundary measurements. In theoretical considerations it is usually assumed that the boundary data consists of the Neumann-to-Dirichlet map; when conducting realworld measurements, the obtainable data is a linear finite-dimensional operator mapping electrode currents onto electrode potentials. In this paper it is shown that when using the complete electrode model to handle electrode measurements, the corresponding current-to-voltage map can be seen as a discrete approximation of the traditional Neumann-to-Dirichlet operator. This approximating link is utilized further in the special case of constant background conductivity with inhomogeneities: It is demonstrated how inclusions with strictly higher or lower conductivities can be characterized by the limit behavior of the range of a boundary operator, determined through electrode measurements, when the electrodes get infinitely small and cover all of the object boundary.
\end{abstract}

Key words. electrical impedance tomography, inverse boundary value problems, electrode models, variational principles

AMS subject classifications. 35R30, 35J25

DOI. $10.1137 / \mathrm{S} 0036139903423303$

1. Introduction. The problem of electrical impedance tomography is as follows: Gather information about the admittance tensor $\sigma$ in the elliptic equation

$$
\nabla \cdot \sigma \nabla u=0 \text { in } \Omega
$$

using measurements of current and potential on the boundary $\partial \Omega$. In mathematical analysis of this problem it is usually assumed that the obtainable data are all possible pairs of Neumann and Dirichlet boundary values, i.e., the linear Neumann-to-Dirichlet map. In particular, all uniqueness and reconstruction results have been formulated using this so-called continuum model (CM) - for more details we refer to the review paper [1]. However, when conducting real-life measurements with electrodes, one can control only the net currents through certain surface patches and measure the corresponding potentials on the electrodes, and so the real-life data consists, essentially, of a finite-dimensional linear electrode current-to-electrode voltage operator.

In this work we model the electrode measurements with the complete electrode model (CEM) [9], which has been shown to predict experimental data reasonably well [9] and also give fairly good numerical reconstructions for both experimental and simulated data [12], [11]. Our first goal is to show that the CEM forward problem can, actually, be seen as a Galerkin approximation of the CM forward problem, meaning that the forward solutions for both of these models can be obtained from the very same variational formulation using different function spaces. As a consequence, the forward solution of CEM, with correctly chosen electrode currents, may be considered an approximation for the forward solution of CM corresponding to a given current

\footnotetext{
${ }^{*}$ Received by the editors February 19, 2003; accepted for publication (in revised form) June 17, 2003; published electronically March 30, 2004.

http://www.siam.org/journals/siap/64-3/42330.html

${ }^{\dagger}$ Institute of Mathematics, Helsinki University of Technology, P. O. Box 1100, FIN-02015 HUT, Finland (nuutti.hyvonen@hut.fi).
} 
distribution - or the other way around - with the correspondence getting better as the electrodes get smaller and cover a larger portion of the object boundary. Section 2 considers these matters.

The second objective is to use the approximating link between the different forward models to modify the factorization method for characterizing inclusions, introduced and justified in [3] for electrical impedance tomography and, earlier, in [7] for inverse scattering, to the framework of the CEM. To be more precise, in section 3 the special case of constant background conductivity with inclusions of strictly higher or lower conductivities is considered. It is demonstrated how the inhomogeneities can be characterized by comparing the boundary values of a dipole-like singular solution and the range of a boundary operator, obtained through electrode measurements, as the electrodes grow in number, get infinitely small, and cover all of the object boundary.

2. Approximation properties of the CEM. In this section we aim to show that the CEM can be seen as a finite element approximation of the CM of impedance tomography. In the first subsection, we will introduce the different forward models and consider some of their basic properties. The second subsection will explain how one can approximate the forward solution of CM by the forward solution of CEM with correctly chosen input currents. In the final subsection, we will survey the resemblance between the current-to-potential boundary maps of CM and CEM.

2.1. Forward models. When performing mathematical analysis of the electrical impedance tomography problem, it is traditionally assumed that one is able to use any input current distribution from Sobolev space $H^{-1 / 2}$ resulting in boundary potentials of class $H^{1 / 2}$. On the other hand, when conducting real-world measurements, one can control only the net currents fed through a finite number of electrodes and measure the corresponding electrode potentials. In particular, one does not know the exact distribution of the current penetrating the object boundary.

2.1.1. Continuum forward model. Let $\Omega \subset \mathbb{R}^{n}, n=2,3$, with a smooth boundary be our open bounded region of interest and let $\sigma: \Omega \rightarrow \mathbb{C}^{n \times n}$ be the corresponding admittance tensor. The forward problem of impedance tomography with continuous boundary measurements is as follows: For $f \in H_{0}^{-1 / 2}(\partial \Omega)$ find $u \in$ $H^{1}(\Omega) / \mathbb{C}$ that satisfies weakly

$$
\nabla \cdot \sigma \nabla u=0 \quad \text { in } \Omega, \quad \nu \cdot \sigma \nabla u=f \quad \text { on } \partial \Omega,
$$

where $\nu$ is the outer unit normal on $\partial \Omega$ and

$$
H_{0}^{-1 / 2}(\partial \Omega)=\left\{v \in H^{-1 / 2}(\partial \Omega) \mid\langle v, \mathbf{1}\rangle_{L^{2}(\partial \Omega)}=0\right\}
$$

where $\langle\phi, \psi\rangle_{L^{2}(\partial \Omega)}=\int_{\partial \Omega} \phi \bar{\psi} d S$ denotes the dual pairing of the spaces $H^{-1 / 2}(\partial \Omega)$ and $H^{1 / 2}(\partial \Omega)$. In what follows, we also shall use this same notation for the $L^{2}$ inner product.

If it is assumed that the admittance tensor $\sigma \in \mathbb{C}^{n \times n}$ satisfies

$$
\operatorname{Re}(\sigma x \cdot \bar{x}) \geq c|x|^{2}, \quad|\sigma x \cdot \bar{x}| \leq C|x|^{2}, \quad c, C>0,
$$

for all $x \in \mathbb{C}^{n}$ almost everywhere in $\Omega$, then forward problem (2.1) has a unique solution that depends continuously on the boundary data.

THeOREM 2.1. Let $f \in H_{0}^{-1 / 2}(\partial \Omega)$ and assume that inequalities (2.2) hold. Then forward problem $(2.1)$ has a unique solution $u \in H^{1}(\Omega) / \mathbb{C}$, for which

$$
\|u\|_{H^{1}(\Omega) / \mathbb{C}} \leq C\|f\|_{H^{-1 / 2}(\partial \Omega)} .
$$


Proof. For proof we refer to [10].

Before we can go any further with our analysis, we need to introduce the trace spaces on subsets of the boundary $\partial \Omega$. Below we will list only the basic definitions; for more information the reader should consult [4] and the references cited therein. For $\Gamma \subset \partial \Omega$ we define

$$
H^{1 / 2}(\Gamma)=\left\{\left.v\right|_{\Gamma} \mid v \in H^{1 / 2}(\partial \Omega)\right\} .
$$

We denote the dual space of $H^{1 / 2}(\Gamma)$ by $\tilde{H}^{-1 / 2}(\Gamma)$, and note that $\tilde{H}^{-1 / 2}(\Gamma)$ can be identified with

$$
H_{\bar{\Gamma}}^{-1 / 2}(\partial \Omega)=\left\{v \in H^{-1 / 2}(\partial \Omega) \mid \operatorname{supp} v \in \bar{\Gamma}\right\} .
$$

In what follows, $\langle\cdot, \cdot\rangle_{L^{2}(\Gamma)}$ will denote either the dual pairing between $\tilde{H}^{-1 / 2}(\Gamma)$ and $H^{1 / 2}(\Gamma)$ or the $L^{2}(\Gamma)$ inner product. Finally, $\tilde{H}_{0}^{-1 / 2}(\Gamma)$ is defined to be the subspace of $\tilde{H}^{-1 / 2}(\Gamma)$ over which the dual evaluation with $\mathbf{1} \in H^{1 / 2}(\Gamma)$ vanishes.

Let us consider briefly the following question. If we are trying to use some given input current pattern $f \in H_{0}^{-1 / 2}(\partial \Omega)$ but we are only able to conduct current through a part of the boundary $\Gamma \subset \partial \Omega$ how much does this imperfection affect the forward solution? To begin with, we must choose how to restrict the current $f$ onto the subset $\Gamma$; using $\left.f\right|_{\Gamma}$ is not usually an option since all the current that goes into the object $\Omega$ must come out. Thus, in order to obtain reasonable currents on $\Gamma$, we define a $L^{2}$-orthogonal projection operator $P_{1}: H_{0}^{-1 / 2}(\partial \Omega) \rightarrow \tilde{H}_{0}^{-1 / 2}(\Gamma) \subset H_{0}^{-1 / 2}(\partial \Omega)$, where the inclusion is achieved through zero continuation, by

$$
P_{1} f=\left.f\right|_{\Gamma}+\frac{1}{|\Gamma|}\langle f, \mathbf{1}\rangle_{L^{2}(\partial \Omega \backslash \bar{\Gamma})} .
$$

Theorem 2.2. Assume that $\sigma \in \mathbb{C}^{n \times n}$ satisfies (2.2), and let $u^{0}$ be the solution of (2.1) corresponding to a given current pattern $f \in H_{0}^{-1 / 2}(\partial \Omega)$. Further, let $u$ be the solution of problem (2.1) associated with the approximating input current $P_{1} f \in$ $H_{0}^{-1 / 2}(\partial \Omega)$. Then we have the estimate

$$
\left\|u^{0}-\left.u\right|_{H^{1}(\Omega) / \mathbb{C}} \leq \frac{C}{|\Gamma|^{1 / 2}}\right\| f \|_{\tilde{H}^{-1 / 2}(\partial \Omega \backslash \bar{\Gamma})},
$$

where $C>0$ can be chosen independently of the geometry of $\Gamma$ as a subset of $\partial \Omega$.

Proof. For $f \in H_{0}^{-1 / 2}(\partial \Omega)$ we have

$$
\begin{aligned}
\left\|f-P_{1} f\right\|_{H^{-1 / 2}(\partial \Omega)} & \leq \frac{1}{|\Gamma|}\left|\langle f, \mathbf{1}\rangle_{L^{2}(\partial \Omega \backslash \bar{\Gamma})}\right|\|\mathbf{1}\|_{\tilde{H}^{-1 / 2}(\Gamma)}+\|f\|_{\tilde{H}^{-1 / 2}(\partial \Omega \backslash \bar{\Gamma})} \\
& \leq\left(\frac{\|\mathbf{1}\|_{H^{1 / 2}(\partial \Omega \backslash \bar{\Gamma})}|| \mathbf{1} \mid \|_{\tilde{H}^{-1 / 2}(\Gamma)}}{|\Gamma|}+1\right)\|f\|_{\tilde{H}^{-1 / 2}(\partial \Omega \backslash \bar{\Gamma})} \\
& \leq \frac{C}{|\Gamma|^{1 / 2}}\|f\|_{\tilde{H}^{-1 / 2}(\partial \Omega \backslash \bar{\Gamma})},
\end{aligned}
$$

where $C>0$ can, clearly, be chosen independently of the geometry of $\Gamma$. Thus, the claim follows by applying Theorem 2.1 to the difference of the solutions $u^{0}-u$.

In a sense the result of Theorem 2.2 is quite natural: The discrepancy in the forward solution is bounded by the norm of the current that we were not able to use. 
2.1.2. Complete electrode forward model. Next, we will introduce the CEM, which has been shown to model real-world electrode measurements reasonably well [9]. Assume that the boundary of the investigated object $\Omega$ is smooth and partially covered with electrodes $e_{m} \subset \partial \Omega, 1 \leq m \leq M$, which are identified by the parts of the surface that they cover and assumed to be ideal conductors. The union of the electrode patches is denoted by $\Gamma_{e}=\cup_{m} e_{m} \subset \partial \Omega$. All electrodes are used for both current injection and voltage measurement, and the current and voltage patterns are denoted by $\left\{I_{m}\right\},\left\{U_{m}\right\} \subset \mathbb{C}, 1 \leq m \leq M$, respectively. To make the model even more flexible, we assume that on $\Gamma_{n} \subset \partial \Omega, \Gamma_{n} \cap \Gamma_{e}=\emptyset$, the current input is given in the continuous sense; i.e., on $\Gamma_{n}$ the data belongs to $\tilde{H}^{-1 / 2}\left(\Gamma_{n}\right)$. Note that this kind of Neumann boundary is not usually included in the formulation of the CEM; here we introduce it to lighten our work load in section 3.

When conducting measurements with electrodes, a thin highly resistive layer is formed at the electrode-object interface [9]. It is characterized by the contact impedance $z: \partial \Omega \rightarrow \mathbb{C}$ that in our framework is assumed to be an integrable function satisfying

$$
\operatorname{Re} z \geq z_{0}>0, \quad|z| \leq z_{1}<\infty,
$$

almost everywhere on $\partial \Omega$. Note that the value of $z$ between the electrodes indicates the fictitious value of the contact impedance, i.e., the value of the contact impedance if an electrode were present.

Traditionally, the electrode currents and potentials are handled as vectors of $\mathbb{C}^{M}$ [9]. However, encouraged by the fact that in CM the boundary potentials and currents are elements of $L^{2}$-based Sobolev spaces, in this work we interpret the electrode currents and potentials as elements of the subspace

$$
T=\left\{V \in L^{2}\left(\Gamma_{e}\right) \mid V=\sum_{m=1}^{M} \chi_{e_{m}} V_{m}, V_{m} \in \mathbb{C}, 1 \leq m \leq M\right\} \subset L^{2}(\partial \Omega) .
$$

In what follows, we will also use the subspace

$$
T_{0}=\left\{V \in T \mid \int_{\partial \Omega} V d S=0\right\} \subset L_{0}^{2}(\partial \Omega),
$$

to which the electrode currents belong if there is no Neumann boundary $\Gamma_{n}$.

With this convention the forward problem corresponding to the CEM is as follows. For input currents $I \in T$ and $g \in \tilde{H}^{-1 / 2}\left(\Gamma_{n}\right)$, with $I+g \in H_{0}^{-1 / 2}(\partial \Omega)$, find $\left(u^{e}, U^{e}\right) \in$ $\left(H^{1}(\Omega) \oplus T\right) / \mathbb{C}$ that satisfies weakly

$$
\begin{gathered}
\nabla \cdot \sigma \nabla u^{e}=0 \text { in } \Omega, \quad \nu \cdot \sigma \nabla u^{e}=0 \text { on } \partial \Omega \backslash\left(\bar{\Gamma}_{e} \cup \bar{\Gamma}_{n}\right), \quad \nu \cdot \sigma \nabla u^{e}=g \text { on } \Gamma_{n}, \\
u^{e}+z \nu \cdot \sigma \nabla u^{e}=U^{e} \text { on } \Gamma_{e}, \quad \frac{1}{\left|e_{m}\right|} \int_{e_{m}} \nu \cdot \sigma \nabla u^{e} d S=I_{m}, \quad 1 \leq m \leq M .
\end{gathered}
$$

Note that the above formulation of the complete electrode forward problem differs from the one in [9] by the scaling factor $1 /\left|e_{m}\right|$ in the last equation of (2.7). However, the underlying physical interpretation stays the same: In [9] the net currents through electrodes were used; here we use the average currents. For more thorough physical justification of (2.7), the reader should consult [9].

Theorem 2.3. Assume that (2.2) and (2.4) hold and let $I \in T$ and $g \in$ $\tilde{H}^{-1 / 2}\left(\Gamma_{n}\right)$, with $I+g \in H_{0}^{-1 / 2}(\partial \Omega)$, be given current patterns. Then problem (2.7) 
has a unique solution $\left(u^{e}, U^{e}\right) \in\left(H^{1}(\Omega) \oplus T\right) / \mathbb{C}$. Further, this solution depends continuously on the data; i.e.,

$$
\inf _{c \in \mathbb{C}}\left\{\left\|u^{e}-c\right\|_{H^{1}(\Omega)}^{2}+\left\|U^{e}-c\right\|_{L^{2}\left(\Gamma_{e}\right)}^{2}\right\}^{1 / 2} \leq C\left\{\|I\|_{L^{2}\left(\Gamma_{e}\right)}+\|g\|_{\tilde{H}^{-1 / 2}\left(\Gamma_{n}\right)}\right\},
$$

where $C>0$ can be chosen independently of the geometry of $\Gamma_{e}$ as a subset of $\partial \Omega$.

By using material in [9], one could easily provide a proof for Theorem 2.3. However, since we have included the Neumann data on $\Gamma_{n}$ in our model and, in addition, we are trying to build a connection between the complete electrode forward problem (2.7) and the continuum forward problem (2.1), we prefer a slightly different working order and postpone the proof until subsection 2.2.

2.2. Approximating with the CEM. In this subsection we aim to show that the CEM can be viewed as a real-world finite element approximation of the mathematically more tractable CM. To be more precise, with the help of the orthogonal projection $P_{2}: L^{2}\left(\Gamma_{e}\right) \rightarrow T$ given by

$$
P_{2} f=\sum_{m=1}^{M} \chi_{e_{m}} \frac{1}{\left|e_{m}\right|} \int_{e_{m}} f d S, \quad f \in L^{2}\left(\Gamma_{e}\right),
$$

we may write the main result of this subsection as follows in Theorem 2.4.

Theorem 2.4. Assume that $\sigma$ and $z$ satisfy (2.2) and (2.4), respectively. Let $f \in$ $H_{0}^{-1 / 2}(\partial \Omega)$, with $\left.f\right|_{\Gamma_{e}} \in L^{2}\left(\Gamma_{e}\right)$, be a given input current and let $u^{0} \in H^{1}(\Omega) / \mathbb{C}$ be the corresponding solution of $(2.1)$. Further, let $\left(u^{e}, U^{e}\right) \in\left(H^{1}(\Omega) \oplus T\right) / \mathbb{C}$ be the unique solution of (2.7) with the input currents $\left.P_{2}\left(P_{1} f\right)\right|_{\Gamma_{e}} \in T$ and $\left.\left(P_{1} f\right)\right|_{\Gamma_{n}} \in \tilde{H}^{-1 / 2}\left(\Gamma_{n}\right)$, where $P_{2}$ is given by (2.9) and $P_{1}$ by (2.3) with $\Gamma=\Gamma_{e} \cup \Gamma_{n}$. Then it holds that

$$
\left\|u^{0}-u^{e}\right\|_{H^{1}(\Omega) / \mathbb{C}} \leq C\left\{\frac{1}{|\Gamma|^{1 / 2}}\|f\|_{\tilde{H}^{-1 / 2}(\partial \Omega \backslash \bar{\Gamma})}+\inf _{V \in T}\left\|U^{0}-V\right\|_{L^{2}\left(\Gamma_{e}\right) / \mathbb{C}}\right\},
$$

where $C>0$ can be chosen independently of the geometry of $\Gamma_{e}$ as a subset of $\partial \Omega$, the subspace $T \subset L^{2}\left(\Gamma_{e}\right)$ is given in (2.5), and $U^{0}=\left.u^{0}\right|_{\Gamma_{e}}+\left.z f\right|_{\Gamma_{e}}$.

Theorem 2.4 tells us, roughly speaking, that for a given current pattern the best correspondence between the solutions of the forward problems (2.1) and (2.7) is obtained when the electrodes are as small as possible and the gaps between the adjacent electrodes are as narrow as possible.

In order to prove Theorem 2.4, we need, first of all, a suitable variational problem: For $f \in L^{2}\left(\Gamma_{e}\right)$ and $g \in \tilde{H}^{-1 / 2}\left(\Gamma_{n}\right)$, with $f+g \in H_{0}^{-1 / 2}(\partial \Omega)$, find $(u, U) \in H=$ $\left(H^{1}(\Omega) \oplus L^{2}\left(\Gamma_{e}\right)\right) / \mathbb{C}$ such that

$$
B((u, U),(v, V))=F(v, V) \text { for all }(v, V) \in H,
$$

where

$$
B((u, U),(v, V))=\int_{\Omega} \sigma \nabla u \cdot \nabla \bar{v} d x+\int_{\Gamma_{e}} \frac{1}{z}(U-u)(\bar{V}-\bar{v}) d S
$$

and

$$
F(v, V)=\int_{\Gamma_{e}} f \bar{V} d S+\int_{\Gamma_{n}} g \bar{v} d S
$$


where the latter term is to be interpreted in the sense of the dual pairing between $\tilde{H}^{-1 / 2}\left(\Gamma_{n}\right)$ and $H^{1 / 2}\left(\Gamma_{n}\right)$. To keep the motivation high, note that variational equation (2.10) is quite similar to the variational formulation of the complete electrode forward problem in [9], the only clear difference being the space from which the solution is sought. We claim that (2.10) has a unique solution with some interesting properties.

Before we can prove the unique solvability of (2.10), we still need to introduce an inner product on $H=\left(H^{1}(\Omega) \oplus L^{2}\left(\Gamma_{e}\right) / \mathbb{C}\right.$, namely,

$$
((u, U),(v, V))_{* H}=\int_{\Omega} \nabla u \cdot \nabla \bar{v} d x+\int_{\Gamma_{e}}(U-u)(\bar{V}-\bar{v}) d S
$$

with the corresponding norm

$$
\|(v, V)\|_{* H}^{2}=((v, V),(v, V))_{* H} .
$$

The following lemma tells us that the above inner product and norm are well defined and concordant with the conventional quotient norm of $H$ given by

$$
\|(v, V)\|_{H}=\inf _{c \in \mathbb{C}}\left\{\|v-c\|_{H^{1}(\Omega)}^{2}+\|V-c\|_{L^{2}\left(\Gamma_{e}\right)}^{2}\right\}^{1 / 2} .
$$

Lemma 2.5. The sesquilinear map $(\cdot, \cdot)_{* H}: H \times H \rightarrow \mathbb{C}$ given by (2.13) defines an inner product which is concordant with the quotient topology of $H=\left(H^{1}(\Omega) \oplus L^{2}\left(\Gamma_{e}\right)\right) /$ C. In consequence, $H$ is a Hilbert space.

Proof. Clearly, $(\cdot, \cdot)_{* H}: H \times H \rightarrow \mathbb{C}$ is well defined and satisfies all the inner product axioms. Hence, the only thing we need to show, in order to prove the claim, is that the usual quotient norm $\|(\cdot, \cdot)\|_{H}$ and the norm $\|(\cdot, \cdot)\|_{* H}$ defined in (2.14) are equivalent.

Let $(v, V) \in H$ be arbitrary. With the help of the trace theorem [5], we may estimate

$$
\begin{aligned}
\|(v, V)\|_{* H} & \leq\|\nabla v\|_{L^{2}(\Omega)}+\|V-v\|_{L^{2}\left(\Gamma_{e}\right)} \\
& \leq\|v-c\|_{H^{1}(\Omega)}+\|v-c\|_{L^{2}\left(\Gamma_{e}\right)}+\|V-c\|_{L^{2}\left(\Gamma_{e}\right)} \\
& \leq C\left\{\|v-c\|_{H^{1}(\Omega)}^{2}+\|V-c\|_{L^{2}\left(\Gamma_{e}\right)}^{2}\right\}^{1 / 2} .
\end{aligned}
$$

Since this holds for every $c \in \mathbb{C}$, we actually have

$$
\|(v, V)\|_{* H} \leq C\|(v, V)\|_{H} .
$$

On the other hand, by using the trace theorem and Poincaré's inequality [5], we get

$$
\begin{aligned}
\|(v, V)\|_{H}^{2} & \leq \inf _{c \in \mathbb{C}}\left\{\|v-c\|_{H^{1}(\Omega)}^{2}+2\|v-c\|_{L^{2}\left(\Gamma_{e}\right)}^{2}\right\}+2\|V-v\|_{L^{2}\left(\Gamma_{e}\right)}^{2} \\
& \leq C \inf _{c \in \mathbb{C}}\|v-c\|_{H^{1}(\Omega)}^{2}+2\|V-v\|_{L^{2}\left(\Gamma_{e}\right)}^{2} \\
& \leq C\|(v, V)\|_{* H}^{2} .
\end{aligned}
$$

Combining this with (2.15) completes the proof.

Corollary 2.6. Assume that (2.2) and (2.4) hold. Then the sesquilinear form $B: H \times H \rightarrow \mathbb{C}$ given in (2.11) is continuous as follows:

$$
|B((u, U),(v, V))| \leq C\|(u, U)\|_{H}\|(v, V)\|_{H}, \quad(u, U),(v, V) \in H,
$$


and coercive as follows:

$$
|B((v, V),(v, V))| \geq c\|(v, V)\|_{H}^{2}, \quad(v, V) \in H,
$$

where the positive constants $c$ and $C$ can be chosen independently of the geometry of $\Gamma_{e}$ as a subset of $\partial \Omega$.

Proof. The claim is a straightforward consequence of Lemma 2.5 together with inequalities (2.2) and (2.4).

Next we aim at showing that the functional $F$ on the right-hand side of (2.10) is continuous. To begin with, we extend the trace theorem for the quotient Sobolev spaces as follows in Lemma 2.7.

Lemma 2.7. The quotient trace map

$$
\operatorname{Tr}: H^{1}(\Omega) / \mathbb{C} \rightarrow H^{1 / 2}(\partial \Omega) / \mathbb{C},\left.\quad v \mapsto v\right|_{\partial \Omega},
$$

is bounded.

Proof. The claim is a straightforward consequence of the traditional trace theorem and Poincaré's inequality.

Lemma 2.8. Let $f \in L^{2}\left(\Gamma_{e}\right)$ and $g \in \tilde{H}^{-1 / 2}\left(\Gamma_{n}\right)$ with $f+g \in H_{0}^{-1 / 2}(\partial \Omega)$. Then the linear functional $F: H \rightarrow \mathbb{C}$ given in (2.12) is well defined and continuous.

Proof. Let us first show that $F: H \rightarrow \mathbb{C}$ is well defined. Consider two representatives $(v, V)$ and $(v+c, V+c)$ of the same equivalence class in $H$. Since $f+g \in H_{0}^{-1 / 2}(\partial \Omega)$, we have

$$
F(v+c, V+c)=\int_{\Gamma_{e}} f \bar{V} d S+\int_{\Gamma_{n}} g \bar{v} d S+\bar{c}\langle f+g, \mathbf{1}\rangle_{L^{2}(\partial \Omega)}=F(v, V) .
$$

Further, by the use of (2.16) and Lemma 2.7, we may estimate, for an arbitrary $(v, V) \in H$,

$$
\begin{aligned}
|F(v, V)| & =\inf _{c \in \mathbb{C}}\left|\int_{\Gamma_{e}} f(\bar{V}+\bar{c}) d S+\int_{\Gamma_{n}} g(\bar{v}+\bar{c}) d S\right| \\
& \leq \inf _{c \in \mathbb{C}}\left\{\|f\|_{L^{2}\left(\Gamma_{e}\right)}\|V+c\|_{L^{2}\left(\Gamma_{e}\right)}+\|g\|_{\tilde{H}^{-1 / 2}\left(\Gamma_{n}\right)}\|v+c\|_{H^{1 / 2}\left(\Gamma_{n}\right)}\right\} \\
& \leq C\left\{\|f\|_{L^{2}\left(\Gamma_{e}\right)}+\|g\|_{\tilde{H}^{-1 / 2}\left(\Gamma_{n}\right)}\right\}\|(v, V)\|_{H}
\end{aligned}
$$

where $C>0$ can be chosen independently of the geometry of $\Gamma_{e}$ as a subset of $\partial \Omega$. This completes the proof.

Now we have introduced enough weaponry to consider the solvability of (2.10).

LEMma 2.9. Assume that (2.2) and (2.4) hold and let $f \in L^{2}\left(\Gamma_{e}\right)$ and $g \in$ $\tilde{H}^{-1 / 2}\left(\Gamma_{n}\right)$, with $f+g \in H_{0}^{-1 / 2}(\partial \Omega)$, be given current patterns. Then variational equation (2.10) has a unique solution $(u, U) \in H=\left(H^{1}(\Omega) \oplus L^{2}\left(\Gamma_{e}\right)\right) / \mathbb{C}$. Further, the first component of this solution, $u \in H^{1}(\Omega) / \mathbb{C}$, is the unique solution of the continuum forward problem (2.1) with the input current $f+g$, and the second component satisfies $U=\left.u\right|_{\Gamma_{e}}+z f$.

Proof. The existence of a unique solution for (2.10) is a straight consequence of the Lax-Milgram lemma [13], Corollary 2.6, and Lemma 2.8.

Let $u^{0} \in H^{1}(\Omega) / \mathbb{C}$ be the unique solution of (2.1) corresponding to the input current $f+g \in H_{0}^{-1 / 2}(\partial \Omega)$ and define $\left(u^{0}, U^{0}\right)=\left(u^{0},\left.u^{0}\right|_{\Gamma_{e}}+z f\right) \in H$. For an 
arbitrary $(v, V) \in H$, it holds that

$$
\begin{aligned}
B\left(\left(u^{0}, U^{0}\right),(v, V)\right) & =\int_{\partial \Omega} \nu \cdot \sigma \nabla u^{0} \bar{v} d S+\int_{\Gamma_{e}} f(\bar{V}-\bar{v}) d S \\
& =\int_{\Gamma_{e}}\left(\nu \cdot \sigma \nabla u^{0}-f\right) \bar{v} d S+\int_{\Gamma_{e}} f \bar{V} d S+\int_{\Gamma_{n}} \nu \cdot \sigma \nabla u^{0} \bar{v} d S \\
& =\int_{\Gamma_{e}} f \bar{V} d S+\int_{\Gamma_{n}} g \bar{v} d S=F(v, V),
\end{aligned}
$$

where we used Green's formula. Thus, $\left(u^{0}, U^{0}\right) \in H$ is a solution to (2.10), which completes the proof.

Now, it is time to return to the complete electrode forward problem. Note that $H^{\prime}=\left(H^{1}(\Omega) \oplus T\right) / \mathbb{C}$, where $T$ is defined by (2.5), is a subspace of $H=\left(H^{1}(\Omega) \oplus\right.$ $\left.L^{2}\left(\Gamma_{e}\right)\right) / \mathbb{C}$. Thus, the variational problem: For $f \in L^{2}\left(\Gamma_{e}\right)$ and $g \in \tilde{H}^{-1 / 2}\left(\Gamma_{n}\right)$, with $f+g \in H_{0}^{-1 / 2}(\partial \Omega)$, find $(u, U) \in H^{\prime}$ so that

$$
B((u, U),(v, V))=F(v, V) \text { for all }(v, V) \in H^{\prime},
$$

where $B$ and $F$ are defined in (2.11) and (2.12), respectively, can be considered a Galerkin approximation for variational problem (2.10). We claim that the unique solution for this approximating variational problem is in fact the unique solution for the complete electrode forward problem (2.7) with a suitable electrode current.

Lemma 2.10. Assume that (2.2) and (2.4) hold and let $f \in L^{2}\left(\Gamma_{e}\right)$ and $g \in$ $\tilde{H}^{-1 / 2}\left(\Gamma_{n}\right)$, with $f+g \in H_{0}^{-1 / 2}(\partial \Omega)$, be given current patterns. Then variational equation (2.18) has a unique solution $\left(u^{e}, U^{e}\right) \in H^{\prime}=\left(H^{1}(\Omega) \oplus T\right) / \mathbb{C}$ which is also the unique solution of the complete electrode forward problem (2.7) corresponding to the input currents $P_{2} f \in T$, where $P_{2}$ is given by (2.9), and $g \in \tilde{H}^{-1 / 2}\left(\Gamma_{n}\right)$.

Proof. By Corollary 2.6 and Lemma 2.8, the sesquilinear form $B: H \times H \rightarrow \mathbb{C}$ is continuous and coercive, and the linear functional $F: H \rightarrow \mathbb{C}$ is continuous. In consequence, the restrictions $B: H^{\prime} \times H^{\prime} \rightarrow \mathbb{C}$ and $F: H^{\prime} \rightarrow \mathbb{C}$ have these same properties. Further, since $H^{\prime}$ is a closed subspace of the Hilbert space $H$, it is also a Hilbert space, and so the unique existence of a solution to (2.18) follows from the Lax-Milgram lemma [13].

To prove that variational problem (2.18) is equivalent to the complete electrode forward problem (2.7) with the electrode current $P_{2} f \in T$, we write the left-hand side of (2.18) componentwise; i.e., for $(u, U),(v, V) \in H^{\prime}$ we have

$$
B((u, U),(v, V))=\int_{\Omega} \sigma \nabla u \cdot \nabla \bar{v} d S+\sum_{m=1}^{M} \int_{e_{m}} \frac{1}{z}\left(U_{m}-u\right)\left(\bar{V}_{m}-\bar{v}\right) d S .
$$

With the same tactic, the right-hand side of (2.18) can be transformed into

$$
F(v, V)=\sum_{m=1}^{M} \int_{e_{m}} f \bar{V}_{m} d S+\int_{\Gamma_{n}} g \bar{v} d S=\sum_{m=1}^{M}\left|e_{m}\right|\left(P_{2} f\right)_{m} \bar{V}_{m}+\int_{\Gamma_{n}} g \bar{v} d S .
$$

With this convention, the claimed equivalence between problems (2.7) and (2.18) follows by the same line of reasoning as in the proof of Proposition 3.1 in [9], with only slight alterations caused by the excess Neumann term on the right-hand side of (2.18). 
Now we have also the means to prove Theorem 2.3.

Proof of Theorem 2.3. For the given current patterns $I \in T \subset L^{2}\left(\Gamma_{e}\right)$ and $g \in$ $\tilde{H}^{-1 / 2}\left(\Gamma_{n}\right)$, with $I+g \in H_{0}^{-1 / 2}(\partial \Omega)$, the unique existence of the solution $\left(u^{e}, U^{e}\right) \in$ $H^{\prime}=\left(H^{1}(\Omega) \oplus T\right) / \mathbb{C}$ for $(2.7)$ follows from the equivalence between problems (2.18) and (2.7), considered in the proof of Lemma 2.10, by choosing $f=I$ in (2.18) and noting that $P_{2} I=I$. Further, by using Corollary 2.6 and equation (2.17), we may estimate

$$
\begin{aligned}
\left\|\left(u^{e}, U^{e}\right)\right\|_{H}^{2} & \leq C\left|B\left(\left(u^{e}, U^{e}\right),\left(u^{e}, U^{e}\right)\right)\right| \\
& =C\left|F\left(u^{e}, U^{e}\right)\right| \\
& \leq C\left\{\|I\|_{L^{2}\left(\Gamma_{e}\right)}+\|g\|_{\tilde{H}^{-1 / 2}\left(\Gamma_{n}\right)}\right\}\left\|\left(u^{e}, U^{e}\right)\right\|_{H},
\end{aligned}
$$

where the functional $F$, defined in (2.12), corresponds to currents $f=I$ and $g$. This completes the proof.

There are a few things worth noticing. First, the solution $\left(u^{e}, U^{e}\right) \in H^{\prime}$ of $(2.7)$ satisfies

$$
\left.\nu \cdot \sigma \nabla u^{e}\right|_{\Gamma_{e}}=\frac{1}{z}\left(U^{e}-\left.u^{e}\right|_{\Gamma_{e}}\right) .
$$

In particular, $\left.\nu \cdot \sigma \nabla u^{e}\right|_{\Gamma_{e}} \in L^{2}\left(\Gamma_{e}\right)$. Second, the correspondence between problems (2.7) and (2.18) gives the complete electrode forward problem a variational formulation: For $I \in T$ and $g \in \tilde{H}^{-1 / 2}\left(\Gamma_{n}\right)$, with $I+g \in H_{0}^{-1 / 2}(\partial \Omega)$, find $\left(u^{e}, U^{e}\right) \in H^{\prime}$ so that

$$
B\left(\left(u^{e}, U^{e}\right),(v, V)\right)=\int_{\Gamma_{e}} I \bar{V} d S+\int_{\Gamma_{n}} g \bar{v} d S
$$

for all $(v, V) \in H^{\prime}$.

Now we have derived the means to approximate the forward solution of the CM (2.1) by the forward solution of the complete electrode problem (2.7) with a correctly chosen electrode current pattern. First we will consider the case when no current is conducted through $\partial \Omega \backslash\left(\bar{\Gamma}_{e} \cup \bar{\Gamma}_{n}\right)$.

TheOrem 2.11. Assume that $\sigma$ and $z$ satisfy (2.2) and (2.4), respectively. Let $f \in H_{0}^{-1 / 2}(\partial \Omega)$, with $\left.f\right|_{\Gamma_{e}} \in L^{2}\left(\Gamma_{e}\right)$ and $\left.f\right|_{\partial \Omega \backslash\left(\bar{\Gamma}_{e} \cup \bar{\Gamma}_{n}\right)}=0$, be a given input current and let $u \in H^{1}(\Omega) / \mathbb{C}$ be the corresponding solution of (2.1). Further, let $\left(u^{e}, U^{e}\right) \in$ $H^{\prime}=\left(H^{1}(\Omega) \oplus T\right) / \mathbb{C}$ be the unique solution of $(2.7)$ with the input currents $P_{2}\left(\left.f\right|_{\Gamma_{e}}\right) \in$ $T$ and $\left.f\right|_{\Gamma_{n}} \in \tilde{H}^{-1 / 2}\left(\Gamma_{n}\right)$, where $P_{2}$ is given in (2.9). Then it holds that

$$
\left\|\left(u-u^{e}, U-U^{e}\right)\right\|_{H} \leq C \inf _{V \in T}\|U-V\|_{L^{2}\left(\Gamma_{e}\right) / \mathbb{C}},
$$

where $C>0$ can be chosen independently of the geometry of $\Gamma_{e}$ as a subset of $\partial \Omega$, the subspace $T \subset L^{2}\left(\Gamma_{e}\right)$ is given in (2.5), and $U=\left.u\right|_{\Gamma_{e}}+\left.z f\right|_{\Gamma_{e}}$.

Proof. To begin with, note that, according to Lemma 2.9, the pair $(u, U) \in H=$ $\left(H^{1}(\Omega) \oplus L^{2}\left(\Gamma_{e}\right)\right) / \mathbb{C}$ satisfies the variational equation

$$
B((u, U),(v, V))=\int_{\Gamma_{e}} f \bar{V} d S+\int_{\Gamma_{n}} f \bar{v} d S \quad \text { for all }(v, V) \in H,
$$

and, on the other hand, Lemma 2.10 tells us that $\left(u^{e}, U^{e}\right)$ satisfies the very same equation with the space $H$ replaced by the subspace $H^{\prime}$. Since the sesquilinear form $B: H \times H \rightarrow \mathbb{C}$ is continuous and coercive, it follows from Cea's lemma [2] that

$$
\left\|\left(u-u^{e}, U-U^{e}\right)\right\|_{H} \leq C \inf _{(v, V) \in H^{\prime}}\|(u-v, U-V)\|_{H} .
$$


Choosing $v=u$ on the right-hand side of (2.20), we obtain

$$
\begin{aligned}
\inf _{(v, V) \in H^{\prime}}\|(u-v, U-V)\|_{H} & \leq \inf _{V \in T} \inf _{c \in \mathbb{C}}\left\{\|c\|_{H^{1}(\Omega)}^{2}+\|(U-V)-c\|_{L^{2}\left(\Gamma_{e}\right)}^{2}\right\}^{1 / 2} \\
& =\inf _{V \in T}\|U-V\|_{L^{2}\left(\Gamma_{e}\right) / \mathbb{C}} .
\end{aligned}
$$

Hence, combining (2.20) and (2.21), the claim follows.

The following corollary also tells us that the normal derivative of the solution to (2.1) can be approximated with the normal derivative of the solution to (2.7).

COROLlary 2.12. Suppose that the assumptions of Theorem 2.11 are valid. Then, using the same notation as in Theorem 2.11, we have the estimate

$$
\left\|f-\nu \cdot \sigma \nabla u^{e}\right\|_{L^{2}\left(\Gamma_{e}\right)}=\left\|\nu \cdot \sigma \nabla u-\nu \cdot \sigma \nabla u^{e}\right\|_{L^{2}\left(\Gamma_{e}\right)} \leq C \inf _{V \in T}\|U-V\|_{L^{2}\left(\Gamma_{e}\right) / \mathbb{C}} .
$$

Proof. Due to the boundary conditions of (2.7) and the way we have defined $U$ in Theorem 2.11, we may estimate

$$
\begin{aligned}
\left\|\nu \cdot \sigma\left(\nabla u-\nabla u^{e}\right)\right\|_{L^{2}\left(\Gamma_{e}\right)} & \leq C\left\|z \nu \cdot \sigma\left(\nabla u-\nabla u^{e}\right)\right\|_{L^{2}\left(\Gamma_{e}\right)} \\
& =C\left\|\left(U-U^{e}\right)-\left(u-u^{e}\right)\right\|_{L^{2}\left(\Gamma_{e}\right)} \\
& \leq C \inf _{c \in \mathbb{C}}\left\{\left\|\left(U-U^{e}\right)-c\right\|_{L^{2}\left(\Gamma_{e}\right)}+\left\|c-\left(u-u^{e}\right)\right\|_{L^{2}\left(\Gamma_{e}\right)}\right\} \\
& \leq C\left\|\left(u-u^{e}, U-U^{e}\right)\right\|_{H},
\end{aligned}
$$

where we took advantage of the trace theorem [5]. The claim follows by combining this with Theorem 2.11.

Finally, it is time to provide a proof for Theorem 2.4 by combining Theorem 2.11 with Theorem 2.2.

Proof of Theorem 2.4. Let $u \in H^{1}(\Omega) / \mathbb{C}$ be the solution to the continuum forward problem (2.1) corresponding to the input current $P_{1} f \in \tilde{H}_{0}^{-1 / 2}(\Gamma)$, where $P_{1}$ is defined by (2.3), and define $U=\left.\left(u+z P_{1} f\right)\right|_{\Gamma_{e}}$. According to Theorems 2.2 and 2.11, we can estimate

$$
\begin{aligned}
\left\|u^{0}-u^{e}\right\|_{H^{1}(\Omega) / \mathbb{C}} & \leq\left\|u^{0}-u\right\|_{H^{1}(\Omega) / \mathbb{C}}+\left\|u-u^{e}\right\|_{H^{1}(\Omega) / \mathbb{C}} \\
& \leq C\left\{\frac{1}{|\Gamma|^{1 / 2}}\|f\|_{\tilde{H}^{-1 / 2}(\partial \Omega \backslash \bar{\Gamma})}+\inf _{V \in T}\|U-V\|_{L^{2}\left(\Gamma_{e}\right) / \mathbb{C}}\right\},
\end{aligned}
$$

where the latter term may be divided into two parts by using the triangle inequality

$$
\inf _{V \in T}\|U-V\|_{L^{2}\left(\Gamma_{e}\right) / \mathbb{C}} \leq \inf _{V \in T}\left\|U^{0}-V\right\|_{L^{2}\left(\Gamma_{e}\right) / \mathbb{C}}+\left\|U^{0}-U\right\|_{L^{2}\left(\Gamma_{e}\right) / \mathbb{C}} .
$$

Further, by using (2.3), (2.4), Lemma 2.7, Theorem 2.2, and the way $U^{0}$ and $U$ are defined, we deduce that

$$
\begin{aligned}
\left\|U^{0}-U\right\|_{L^{2}\left(\Gamma_{e}\right) / \mathbb{C}} & \leq\left\|u^{0}-u\right\|_{L^{2}\left(\Gamma_{e}\right) / \mathbb{C}}+\left\|z\left(f-P_{1} f\right)\right\|_{L^{2}\left(\Gamma_{e}\right) / \mathbb{C}} \\
& \leq C\left\|u^{0}-u\right\|_{H^{1}(\Omega) / \mathbb{C}}+\frac{1}{|\Gamma|}\left|\langle f, \mathbf{1}\rangle_{L^{2}(\partial \Omega \backslash \bar{\Gamma})}\right|\|z\|_{L^{2}\left(\Gamma_{e}\right) / \mathbb{C}} \\
& \leq\left\{\frac{C}{|\Gamma|^{1 / 2}}+\frac{z_{1}}{|\Gamma|}\|\mathbf{1}\|_{H^{1 / 2}(\partial \Omega \backslash \bar{\Gamma})}\|\mathbf{1}\|_{L^{2}\left(\Gamma_{e}\right)}\right\}\|f\|_{\tilde{H}^{-1 / 2}(\partial \Omega \backslash \bar{\Gamma})} \\
& \leq \frac{C}{|\Gamma|^{1 / 2}}\|f\|_{\tilde{H}^{-1 / 2}(\partial \Omega \backslash \bar{\Gamma})} .
\end{aligned}
$$

The claim follows by combining (2.22), (2.23), and (2.24). 
2.3. Comparing current-to-voltage maps. It is time to move on to consider the current-to-potential maps corresponding to CM and CEM. In order to keep things simple, in this subsection we assume that there is no Neumann boundary; the introduction of $\Gamma_{n}$ in (2.7) was just a technical detail that is useful for us in the next section-usually, it does not play any part in real-world measurements. As a further simplification, we assume that all used current patterns are square integrable.

When dealing with the inverse problem for the CM, it is usually assumed that the known data is the linear Neumann-to-Dirichlet map, i.e., the operator that maps the applied current pattern onto the boundary potential

$$
\Lambda_{\sigma}:\left.f \mapsto u_{\sigma}^{0}\right|_{\partial \Omega}
$$

which is isomorphic from $H_{0}^{-1 / 2}(\partial \Omega)$ onto $H^{1 / 2}(\partial \Omega) / \mathbb{C} \sim H_{0}^{1 / 2}(\partial \Omega)$ and depends nonlinearly on $\sigma$. On the other hand, when conducting real-life measurements with the CEM, the only information one is able to obtain is the linear relation between the applied average currents $I_{m} \in \mathbb{C}, 1 \leq m \leq M$, and the electrode voltages $U_{m}^{e} \in \mathbb{C}$, $1 \leq m \leq M$, given by

$$
R_{\sigma} I=U^{e},
$$

where $R_{\sigma}: T_{0} \rightarrow T / \mathbb{C}$ can be expressed in matrix form since $T_{0}, T / \mathbb{C} \sim \mathbb{C}^{M-1}$. The next challenge is to build some kind of approximating link between the operators $\Lambda_{\sigma}$ and $R_{\sigma}$.

Assume that there is no Neumann boundary; i.e., $\Gamma_{n}=\emptyset$ in (2.7). By combining $R_{\sigma}$ with the projection

$$
P=P_{2} P_{1}: L_{0}^{2}(\partial \Omega) \rightarrow T_{0},
$$

where $T_{0}$ is given by (2.6), and the projections $P_{1}$, with $\Gamma=\Gamma_{e}$, and $P_{2}$ are defined in (2.3) and (2.9), respectively, we get the map

$$
R_{\sigma} P: L_{0}^{2}(\partial \Omega) \rightarrow T / \mathbb{C}, \quad f \mapsto U_{\sigma}^{e}=\left.\left(u_{\sigma}^{e}+z \nu \cdot \sigma \nabla u_{\sigma}^{e}\right)\right|_{\Gamma_{e}},
$$

where $\left(u_{\sigma}^{e}, U_{\sigma}^{e}\right) \in\left(H^{1}(\Omega) \oplus T\right) / \mathbb{C}$ is the solution of $(2.7)$ corresponding to the electrode current $\operatorname{Pf}$ and the admittance $\sigma$.

The resemblance between the operators $\Lambda_{\sigma}$ and $R_{\sigma} P$ is quite apparent. However, $R_{\sigma} P$ is not a pure current-to-voltage map, which prevents us from using Theorem 2.4 to investigate the situation further. Luckily, in many of the reconstruction algorithms for the CM, one does not use merely $\Lambda_{\sigma}$ but the difference [1]

$$
\Lambda_{\sigma}-\Lambda_{\mathbf{1}}:\left.f \mapsto\left(u_{\sigma}^{0}-u_{\mathbf{1}}^{0}\right)\right|_{\partial \Omega},
$$

where $\Lambda_{\mathbf{1}}$ is the Neumann-to-Dirichlet map corresponding to the unit admittance distribution, and $u_{1}^{0} \in H^{1}(\Omega) / \mathbb{C}$ is the associated forward solution for the input current $f \in L_{0}^{2}(\partial \Omega)$. For the complete electrode counterpart, we get the formula

$$
\left(R_{\sigma}-R_{\mathbf{1}}\right) P:\left.f \mapsto\left(u_{\sigma}^{e}-u_{\mathbf{1}}^{e}+z \nu \cdot\left(\sigma \nabla u_{\sigma}^{e}-\nabla u_{\mathbf{1}}^{e}\right)\right)\right|_{\Gamma_{e}},
$$

which is, actually, quite close to (2.27).

THEOREM 2.13. Assume that $\sigma$ and $z$ satisfy (2.2) and (2.4), respectively, and let $f \in L_{0}^{2}(\partial \Omega)$ be a given current pattern. It holds that

$$
C\left\{\frac{1}{\left|\Gamma_{e}\right|^{1 / 2}}\|f\|_{\tilde{H}-1 / 2\left(\partial \Omega \backslash \bar{\Gamma}_{e}\right)}+\inf _{V \in T}\left\|U_{\sigma}^{0}-V\right\|_{L^{2}\left(\Gamma_{e}\right) / \mathbb{C}}+\inf _{V \in T}\left\|U_{1}^{0}-V\right\|_{L^{2}\left(\Gamma_{e}\right) / \mathbb{C}}\right\},
$$


where the boundary operators are defined by (2.27) and (2.28), and $U_{\sigma}^{0}=\left.u_{\sigma}^{0}\right|_{\Gamma_{e}}+\left.z f\right|_{\Gamma_{e}}$, $U_{1}^{0}=\left.u_{1}^{0}\right|_{\Gamma_{e}}+\left.z f\right|_{\Gamma_{e}}$, where $u_{\sigma}^{0}$ and $u_{1}^{0}$ are the solutions of the continuum forward problem (2.1) corresponding to the input current $f$ and the impedance tensors $\sigma$ and $\mathbf{1}$, respectively.

Proof. With the help of (2.27) and (2.28), the left-hand side of (2.29) can be divided into three parts as follows:

$$
\begin{aligned}
& \left\|\left(\left(\Lambda_{\sigma}-\Lambda_{\mathbf{1}}\right)-\left(R_{\sigma}-R_{\mathbf{1}}\right) P\right) f\right\|_{L^{2}\left(\Gamma_{e}\right) / \mathbb{C}} \leq\left\|u_{\sigma}^{0}-u_{\sigma}^{e}\right\|_{L^{2}\left(\Gamma_{e}\right) / \mathbb{C}}+\left\|u_{\mathbf{1}}^{0}-u_{\mathbf{1}}^{e}\right\|_{L^{2}\left(\Gamma_{e}\right) / \mathbb{C}} \\
& +\left\|z \nu \cdot\left(\sigma \nabla u_{\sigma}^{e}-\nabla u_{1}^{e}\right)\right\|_{L^{2}\left(\Gamma_{e}\right) / \mathbb{C}} \cdot
\end{aligned}
$$

For the first term on the right-hand side of (2.30), it follows from Lemma 2.7 and Theorem 2.4 that

$$
\left\|u_{\sigma}^{0}-u_{\sigma}^{e}\right\|_{L^{2}\left(\Gamma_{e}\right) / \mathbb{C}} \leq C\left\{\frac{1}{\left|\Gamma_{e}\right|^{1 / 2}}\|f\|_{\tilde{H}^{-1 / 2}\left(\partial \Omega \backslash \bar{\Gamma}_{e}\right)}+\inf _{V \in T}\left\|U_{\sigma}^{0}-V\right\|_{L^{2}\left(\Gamma_{e}\right) / \mathbb{C}}\right\} .
$$

By the same means, we get an exactly similar estimate for the second term on the right-hand side of (2.30).

In order to handle the third term on the right-hand side of (2.30), let $u_{\sigma}, u_{\mathbf{1}} \in$ $H^{1}(\Omega) / \mathbb{C}$ be the solutions of $(2.1)$ with the input current $P_{1} f \in L_{0}^{2}\left(\Gamma_{e}\right)$, where $P_{1}$ : $L_{0}^{2}(\partial \Omega) \rightarrow L_{0}^{2}\left(\Gamma_{e}\right)$ is defined by $(2.3)$ with $\Gamma=\Gamma_{e}$, for the admittances $\sigma$ and $\mathbf{1}$, respectively, and define $U_{\sigma}=\left.u_{\sigma}\right|_{\Gamma_{e}}+z P_{1} f$ and $U_{\mathbf{1}}=\left.u_{\mathbf{1}}\right|_{\Gamma_{e}}+z P_{1} f$. We use Corollary 2.12 to estimate

$$
\begin{aligned}
\left\|z \nu \cdot\left(\sigma \nabla u_{\sigma}^{e}-\nabla u_{\mathbf{1}}^{e}\right)\right\|_{L^{2}\left(\Gamma_{e}\right) / \mathbb{C}} \leq & \left\|z\left(\nu \cdot \sigma \nabla u_{\sigma}^{e}-P_{1} f\right)\right\|_{L^{2}\left(\Gamma_{e}\right)}+\left\|z\left(P_{1} f-\nu \cdot \nabla u_{\mathbf{1}}^{e}\right)\right\|_{L^{2}\left(\Gamma_{e}\right)} \\
\leq & C\left\{\inf _{V \in T}\left\|U_{\sigma}-V\right\|_{L^{2}\left(\Gamma_{e}\right) / \mathbb{C}}+\inf _{V \in T}\left\|U_{\mathbf{1}}-V\right\|_{L^{2}\left(\Gamma_{e}\right) / \mathbb{C}}\right\} \\
\leq & C\left\{\frac{1}{\left|\Gamma_{e}\right|^{1 / 2}}\|f\|_{\tilde{H}^{-1 / 2}\left(\partial \Omega \backslash \bar{\Gamma}_{e}\right)}+\inf _{V \in T}\left\|U_{\sigma}^{0}-V\right\|_{L^{2}\left(\Gamma_{e}\right) / \mathbb{C}}\right. \\
& \left.+\inf _{V \in T}\left\|U_{\mathbf{1}}^{0}-V\right\|_{L^{2}\left(\Gamma_{e}\right) / \mathbb{C}}\right\},
\end{aligned}
$$

where we also use assumption (2.4) and inequality (2.24) from the proof of Theorem 2.4. The claim follows by combining the estimates for the terms on the right-hand side of (2.30).

Again it is advisable to note a couple of things. First, the above theorem could also have been formulated for currents $f \in H_{0}^{-1 / 2}(\partial \Omega)$ with $\left.f\right|_{\Gamma_{e}} \in L^{2}\left(\Gamma_{e}\right)$; the notation would have been even more cumbersome, however. Second, the images of the boundary maps are compared only on $\Gamma_{e} \subset \partial \Omega$ since in a real-life measurement situation one is not measuring anything outside the electrodes and, thus, there is nothing to compare on $\partial \Omega \backslash \bar{\Gamma}_{e}$. Third, the correspondence between the maps $\Lambda_{\sigma}-\Lambda_{\mathbf{1}}$ and $R_{\sigma}-R_{1}$ gets better when the area covered by the electrodes gets larger and the electrodes get smaller.

3. Characterizing inclusions. In this section we demonstrate how the boundary map $R_{\sigma}-R_{1}$, considered in the previous subsection, can be used to characterize an inclusion $D \subset \Omega$ with conductivity significantly higher or lower than the constant background conductivity. The section is organized as follows. We begin by introducing our framework and listing some basic properties of $R_{\sigma}$. Section 3.1 presents a factorization of $R_{\sigma}-R_{1}$ into three parts. In section 3.2 the operators needed in the 
factorization are investigated further and, finally, in section 3.3 we provide the characterization for the inclusion. This work can be seen as a discrete version of article [3]; also, the mathematical methods used here resemble to some extent those in [3].

Our object $\Omega \subset \mathbb{R}^{n}, n=2,3$, is assumed to be isotropic with a conductivity $0<\sigma \leq C$ and a smooth boundary, and the used input current is assumed to be nonvariating in time. Further, we assume that the contact impedance $z: \partial \Omega \rightarrow \mathbb{R}$ is strictly positive and bounded. Let the subspaces $T \subset L^{2}(\partial \Omega)$ and $T_{0} \subset L_{0}^{2}(\partial \Omega)$ be defined by (2.5) and (2.6), respectively, with the multiplier field $\mathbb{C}$ replaced by $\mathbb{R}$. Then, according to Theorem 2.3, for every $I \in T_{0}$ the complete electrode forward problem

$$
\begin{gathered}
\nabla \cdot \sigma \nabla u=0 \text { in } \Omega, \quad \nu \cdot \sigma \nabla u=0 \text { on } \partial \Omega \backslash \bar{\Gamma}_{e}, \\
u+z \nu \cdot \sigma \nabla u=U \text { on } \Gamma_{e}, \quad \frac{1}{\left|e_{m}\right|} \int_{e_{m}} \nu \cdot \sigma \nabla u d S=I_{m}, \quad 1 \leq m \leq M,
\end{gathered}
$$

has a unique solution $(u, U) \in H^{1}(\Omega) \oplus T_{0}$, where we have specified the ground level of the potential in an obvious way. The corresponding boundary map $R_{\sigma}: T_{0} \rightarrow T_{0}$ is defined through $R_{\sigma} I=U$.

We emphasize the resemblance between $R_{\sigma}$ and its continuous counterpart $\Lambda_{\sigma}$, given in (2.25), by showing that $R_{\sigma}$ inherits some basic characteristics of $\Lambda_{\sigma}$.

Lemma 3.1. The operator $R_{\sigma}: T_{0} \rightarrow T_{0}$ is self-adjoint and positive. Furthermore, $R_{\sigma}$ is monotonically decreasing; i.e.,

$$
\left\langle I, R_{\sigma} I\right\rangle_{L^{2}(\partial \Omega)}>\left\langle I, R_{\tilde{\sigma}} I\right\rangle_{L^{2}(\partial \Omega)},
$$

for $\sigma \leq \tilde{\sigma}, \sigma \neq \tilde{\sigma}$ on a set of nonzero measure, and $I \neq 0$.

Proof. The result follows by imitating the proof of Lemma 2.1 in [3] with the help of the weak formulation of (3.1) given by (2.19).

Since $T_{0}$ is a finite-dimensional subspace of $L_{0}^{2}(\partial \Omega)$, the monotonicity property of Lemma 3.1 implies that $R_{\sigma}-R_{\tilde{\sigma}}: T_{0} \rightarrow T_{0}$ has a bounded inverse if the assumptions of Lemma 3.1 are valid.

Corollary 3.2. Let $\sigma \leq \tilde{\sigma}$, and $\sigma \neq \tilde{\sigma}$ on a set of nonzero measure. Then $R_{\sigma}-R_{\tilde{\sigma}}: T_{0} \rightarrow T_{0}$ is strictly positive. In particular, $R_{\sigma}-R_{\tilde{\sigma}}$ is bijective and has a bounded inverse.

Proof. From the monotonicity property of Lemma 3.1 we straight away obtain

$$
\left\langle I,\left(R_{\sigma}-R_{\tilde{\sigma}}\right) I\right\rangle_{L^{2}(\partial \Omega)}>0
$$

for every $I \in T_{0}, I \neq 0$. Since $T_{0}$ is finite-dimensional and $R_{\sigma}-R_{\tilde{\sigma}}$ is linear, this induces the estimate

$$
\left\langle I,\left(R_{\sigma}-R_{\tilde{\sigma}}\right) I\right\rangle_{L^{2}(\partial \Omega)} \geq c\|I\|_{L^{2}(\partial \Omega)}^{2}, \quad c>0 .
$$

The injectivity, or, equivalently, the bijectivity, of $R_{\sigma}-R_{\tilde{\sigma}}: T_{0} \rightarrow T_{0}$ follows trivially from (3.2), which completes the proof.

3.1. Factorization of $\boldsymbol{R}_{\boldsymbol{\sigma}}-\boldsymbol{R}_{\mathbf{1}}$. From now on we assume that the conductivity inside $\Omega$ is of the form

$$
\sigma= \begin{cases}\kappa & \text { in } D \\ 1 & \text { in } \Omega \backslash \bar{D}\end{cases}
$$


where $\kappa \neq 1$ is a positive constant and $D$ is an open connected subset of $\Omega$ with a smooth connected boundary and $\partial D \cap \partial \Omega=\emptyset$. Our aim is to prove the following theorem.

THEOREM 3.3. Assume that the conductivity inside $\Omega$ is of the form given in (3.3). Then the difference of the boundary maps $R_{\sigma}, R_{1}: T_{0} \rightarrow T_{0}$ can be factorized as $R_{\sigma}-R_{1}=L F L^{\prime}$, where $L: H_{0}^{-1 / 2}(\partial D) \rightarrow T_{0}$ is continuous and surjective, its adjoint operator $L^{\prime}: T_{0} \rightarrow H_{0}^{1 / 2}(\partial D)$ is continuous and injective, and $F: H_{0}^{1 / 2}(\partial D) \rightarrow$ $H_{0}^{-1 / 2}(\partial D)$ is self-adjoint, bijective, and either positive or negative definite.

Before we can introduce the operators needed for the above factorization, we must consider some notational details. On the inner boundary $\partial D$ we define

$$
v^{ \pm}(x)=\lim _{t \rightarrow 0^{+}} v(x \pm t \nu) \quad \text { and } \quad \frac{\partial v^{ \pm}}{\partial \nu}(x)=\lim _{t \rightarrow 0^{+}} \nu \cdot \nabla v(x \pm t \nu),
$$

for $x \in \partial D$ with $\nu(x)$ the unit normal pointing out of $D$, and further,

$$
[v]_{\partial D}=v^{+}-v^{-} \text {and }\left[\sigma \frac{\partial v}{\partial \nu}\right]_{\partial D}={\frac{\partial v^{+}}{\partial \nu}}^{+}-\kappa{\frac{\partial v^{-}}{\partial \nu}}^{-}
$$

Let us now define $L$ and $L^{\prime}$. By replacing $\Omega$ with $\Omega \backslash \bar{D}$ and choosing $\Gamma_{n}=\partial D$ in (2.7) and Theorem 2.3, we note that for every $\phi \in H_{0}^{-1 / 2}(\partial D)$ the boundary value problem

$$
\begin{gathered}
\Delta v=0 \text { in } \Omega \backslash \bar{D}, \quad \frac{\partial v}{\partial \nu}=0 \text { on } \partial \Omega \backslash \bar{\Gamma}_{e}, \quad \frac{\partial v^{+}}{\partial \nu}=\phi \text { on } \partial D \\
v+z \frac{\partial v}{\partial \nu}=V \text { on } \Gamma_{e}, \quad \frac{1}{\left|e_{m}\right|} \int_{e_{m}} \frac{\partial v}{\partial \nu} d S=0, \quad 1 \leq m \leq M
\end{gathered}
$$

has a unique solution $(v, V) \in H^{1}(\Omega \backslash \bar{D}) \oplus T_{0}$, where we have fixed the ground level of the potential. Thus, we may define the operator $L$ by

$$
L: H_{0}^{-1 / 2}(\partial D) \rightarrow T_{0}, \quad \phi \mapsto V .
$$

With $I^{\prime} \in T_{0}$, let us next consider the boundary value problem

$$
\begin{aligned}
& \Delta v^{\prime}=0 \text { in } \Omega \backslash \bar{D}, \quad \frac{\partial v^{\prime}}{\partial \nu}=0 \quad \text { on } \partial \Omega \backslash \bar{\Gamma}_{e}, \quad \frac{\partial v^{\prime+}}{\partial \nu}=0 \quad \text { on } \partial D, \\
& v^{\prime}+z \frac{\partial v^{\prime}}{\partial \nu}=V^{\prime} \quad \text { on } \Gamma_{e}, \quad \frac{1}{\left|e_{m}\right|} \int_{e_{m}} \frac{\partial v^{\prime}}{\partial \nu} d S=-I_{m}^{\prime}, \quad 1 \leq m \leq M,
\end{aligned}
$$

which, according to Theorem 2.3, also has a unique solution $\left(v^{\prime}, V^{\prime}\right) \in H_{0, \partial D}^{1}(\Omega \backslash \bar{D}) \oplus$ $T$, where

$$
H_{0, \partial D}^{1}(\Omega \backslash \bar{D})=\left\{u \in H^{1}(\Omega \backslash \bar{D}) \mid \int_{\partial D} u d S=0\right\} .
$$

We define $L^{\prime}$ by

$$
L^{\prime}: T_{0} \rightarrow H_{0}^{1 / 2}(\partial D),\left.\quad I^{\prime} \mapsto v^{\prime}\right|_{\partial D}
$$

The following lemma shows that $L$ and $L^{\prime}$ are bounded and adjoint, and have the mapping properties advertised above. 
Lemma 3.4. The operators $L: H_{0}^{-1 / 2}(\partial D) \rightarrow T_{0}$ and $L^{\prime}: T_{0} \rightarrow H_{0}^{1 / 2}(\partial D)$ defined by (3.5) and (3.8), respectively, are bounded (independently of the geometry of $\left.\Gamma_{e}\right)$ and adjoint. Further, $L$ is surjective and $L^{\prime}$ is injective.

Proof. We begin with the boundedness of $L$. For $\phi \in H_{0}^{-1 / 2}(\partial D)$ let $(v, V) \in$ $H^{1}(\Omega \backslash \bar{D}) \oplus T_{0}$ be the unique solution of (3.4), suggested by Theorem 2.3. From the continuous dependence on the data (2.8) and since $V \in T_{0} \subset L_{0}^{2}\left(\Gamma_{e}\right)$, it follows that

$$
\|V\|_{L^{2}\left(\Gamma_{e}\right)}=\|V\|_{L^{2}\left(\Gamma_{e}\right) / \mathbb{R}} \leq\|(v, V)\|_{\left(H^{1}(\Omega \backslash \bar{D}) \oplus L^{2}\left(\Gamma_{e}\right)\right) / \mathbb{R}} \leq C\|\phi\|_{H^{-1 / 2}(\partial D)},
$$

which proves the continuity of $L: H_{0}^{-1 / 2}(\partial D) \rightarrow T_{0}$.

Next we shall prove that $L^{\prime}: T_{0} \rightarrow H_{0}^{1 / 2}(\partial D)$ is the adjoint of $L$. Let $(v, V) \in$ $H^{1}(\Omega \backslash \bar{D}) \oplus T_{0}$ and $\left(v^{\prime}, V^{\prime}\right) \in H_{0, \partial D}^{1}(\Omega \backslash \bar{D}) \oplus T$ be the unique solutions of (3.4) and (3.6), respectively. Then it holds that

$$
\begin{aligned}
\left\langle I^{\prime}, L \phi\right\rangle_{L^{2}(\partial \Omega)} & =\int_{\Gamma_{e}}\left(I^{\prime}+\frac{\partial v^{\prime}}{\partial \nu}\right) V d S-\int_{\Gamma_{e}} \frac{\partial v^{\prime}}{\partial \nu} V d S \\
& =-\int_{\Gamma_{e}} \frac{\partial v^{\prime}}{\partial \nu} V d S=-\int_{\Gamma_{e}} \frac{\partial v^{\prime}}{\partial \nu}\left(v+z \frac{\partial v}{\partial \nu}\right) d S \\
& =-\int_{\Gamma_{e}} \frac{\partial v^{\prime}}{\partial \nu} v d S-\int_{\Gamma_{e}}\left(z \frac{\partial v^{\prime}}{\partial \nu}-V^{\prime}\right) \frac{\partial v}{\partial \nu} d S \\
& =-\int_{\Gamma_{e}} \frac{\partial v^{\prime}}{\partial \nu} v d S+\int_{\Gamma_{e}} v^{\prime} \frac{\partial v}{\partial \nu} d S \\
& =-\int_{\partial D} \frac{\partial v^{\prime}}{\partial \nu} v d S+\int_{\partial D} v^{\prime} \frac{\partial v^{+}}{\partial \nu} d S=\left\langle L^{\prime} I^{\prime}, \phi\right\rangle_{L^{2}(\partial D)},
\end{aligned}
$$

where we used the boundary conditions that the pairs $(v, V)$ and $\left(v^{\prime}, V^{\prime}\right)$ satisfy together with Green's formula. Since $L$ is bounded and $L^{\prime}$ is its adjoint operator, $L^{\prime}$ is also bounded.

The injectivity of $L^{\prime}$ is easy to obtain: Let $I^{\prime} \in T_{0}$ be such that $L^{\prime} I^{\prime}=\left.v^{\prime}\right|_{\partial D}=0$, which means, according to (3.6), that the Cauchy data of $v^{\prime}$ vanishes on $\partial D$. Since $v^{\prime}$ is harmonic in $\Omega \backslash \bar{D}$, this implies that $v^{\prime}=0$ from which it also follows that $I^{\prime}=0$. In addition, due to the finite-dimensionality of $\mathcal{R}(L)$, we have $T_{0}=\mathcal{N}\left(L^{\prime}\right)^{\perp}=\overline{\mathcal{R}(L)}=$ $\mathcal{R}(L)$, which proves the surjectivity of $L$. This completes the proof.

Last but not least, let us introduce $F: H_{0}^{1 / 2}(\partial D) \rightarrow H_{0}^{-1 / 2}(\partial D)$. Let $\psi \in$ $H_{0}^{1 / 2}(\partial D)$ and assume that $\left(w_{\sigma}, W_{\sigma}\right) \in\left(H^{1}(\Omega \backslash \partial D) \oplus T\right) / \mathbb{R}$ is the solution of the diffraction problem

$$
\begin{gathered}
\Delta w=0 \quad \text { in } \Omega \backslash \partial D, \quad \frac{\partial w}{\partial \nu}=0 \quad \text { on } \partial \Omega \backslash \bar{\Gamma}_{e}, \quad w+z \frac{\partial w}{\partial \nu}=W \quad \text { on } \Gamma_{e} \\
{[w]_{\partial D}=\psi, \quad\left[\sigma \frac{\partial w}{\partial \nu}\right]_{\partial D}=0, \quad \frac{1}{\left|e_{m}\right|} \int_{e_{m}} \frac{\partial w}{\partial \nu} d S=0, \quad 1 \leq m \leq M .}
\end{gathered}
$$

We define $F$ by the mapping rule $\left.\psi \mapsto{\frac{\partial\left(w_{\sigma}-w_{1}\right)}{\partial \nu}}^{+}\right|_{\partial D}$, where $w_{1}$ is the solution of (3.9) with $\sigma$ replaced by the unit conductivity 1.

Because the outer boundary condition of (3.9) is not of standard form, one must convince oneself that $w_{\sigma}$ and $w_{1}$, and thereby $F: H_{0}^{1 / 2}(\partial D) \rightarrow H_{0}^{-1 / 2}(\partial D)$, are actually well defined. The following two technical lemmas and a corollary answer all the necessary questions. 
LEMma 3.5. For $\psi \in H_{0}^{1 / 2}(\partial D)$, diffraction problem (3.9) has a unique solution $\left(w_{\sigma}, W_{\sigma}\right) \in\left(H^{1}(\Omega \backslash \partial D) \oplus T\right) / \mathbb{R}$. Further,

$$
\left\|\frac{\partial w_{\sigma}^{+}}{\partial \nu}\right\|_{H^{-1 / 2}(\partial D)} \leq C\|\psi\|_{H^{1 / 2}(\partial D)},
$$

where $C>0$ is independent of the geometry of $\Gamma_{e}$ as a subset of $\partial \Omega$.

Proof. To start with, note that the corresponding traditional diffraction problem

$$
\begin{gathered}
\Delta w=0 \text { in } \Omega \backslash \partial D, \quad \frac{\partial w}{\partial \nu}=0 \text { on } \partial \Omega \\
{[w]_{\partial D}=\psi, \quad\left[\sigma \frac{\partial w}{\partial \nu}\right]_{\partial D}=0}
\end{gathered}
$$

has a unique solution $w_{0} \in H_{0, \partial \Omega}^{1}(\Omega \backslash \partial D)$ (cf. [8]), where the space is defined in equivalence with (3.7). Encouraged by this, we consider the following boundary value problem:

$$
\begin{gathered}
\nabla \cdot \sigma \nabla w=0 \text { in } \Omega, \quad \frac{\partial w}{\partial \nu}=0 \text { on } \partial \Omega \backslash \bar{\Gamma}_{e}, \\
w+z \frac{\partial w}{\partial \nu}=W-w_{0} \text { on } \Gamma_{e}, \quad \frac{1}{\left|e_{m}\right|} \int_{e_{m}} \frac{\partial w}{\partial \nu} d S=0, \quad 1 \leq m \leq M,
\end{gathered}
$$

and try to show that it has a unique solution $\left(w_{e}, W_{e}\right) \in\left(H^{1}(\Omega) \oplus T\right) / \mathbb{R}$.

From considerations in section 2.2 , it follows in a straightforward manner that problem (3.11) is equivalent to the variational problem

$$
B((w, W),(v, V))=\int_{\Gamma_{e}} \frac{1}{z} w_{0}(V-v) d S,
$$

for all $(v, V) \in\left(H^{1}(\Omega) \oplus T\right) / \mathbb{R}$, where the bilinear form $B$ is defined in (2.11). Since, according to Corollary 2.6, $B:\left(H^{1}(\Omega) \oplus T\right) / \mathbb{R} \times\left(H^{1}(\Omega) \oplus T\right) / \mathbb{R} \rightarrow \mathbb{R}$ is continuous and coercive, and the right-hand side of (3.12) clearly defines a continuous linear functional on $\left(H^{1}(\Omega) \oplus T\right) / \mathbb{R}$, equation (3.12) has a unique solution $\left(w_{e}, W_{e}\right) \in\left(H^{1}(\Omega) \oplus T\right) / \mathbb{R}$ due to the Lax-Milgram lemma [13]. Now, it is easy to see that $\left(w_{0}+w_{e}, W_{e}\right) \in$ $\left(H^{1}(\Omega \backslash \partial D) \oplus T\right) / \mathbb{R}$ satisfies the electrode diffraction problem given in (3.9) because of the continuity conditions that $w_{e}$ and $\sigma \frac{\partial w_{e}}{\partial \nu}$ must satisfy on $\partial D$ [8]. In particular, (3.9) has at least one solution.

Assume now that $\left(w_{\sigma}, W_{\sigma}\right) \in\left(H^{1}(\Omega \backslash \partial D) \oplus T\right) / \mathbb{R}$ is a solution of diffraction problem (3.9) corresponding to $\psi \in H_{0}^{1 / 2}(\partial D)$. Then, due to Green's formula and positivity of $z, w_{\sigma}$ satisfies

$$
\begin{aligned}
\left\|\sigma^{1 / 2} \nabla w_{\sigma}\right\|_{L^{2}(\Omega)}^{2} & =\int_{\partial D} w_{\sigma}^{-} \kappa{\frac{w_{\sigma}}{\partial \nu}}^{-} d S-\int_{\partial D} w_{\sigma}^{+}{\frac{\partial w_{\sigma}}{\partial \nu}}^{+} d S+\int_{\partial \Omega} w_{\sigma} \frac{\partial w_{\sigma}}{\partial \nu} d S \\
& =\int_{\partial D}\left(w_{\sigma}^{-}-w_{\sigma}^{+}\right){\frac{\partial w_{\sigma}^{+}}{\partial \nu}}^{+} d S+\int_{\Gamma_{e}}\left(W_{\sigma}-z \frac{\partial w_{\sigma}}{\partial \nu}\right) \frac{\partial w_{\sigma}}{\partial \nu} d S \\
& =-\int_{\partial D} \psi{\frac{\partial w_{\sigma}}{\partial \nu}}^{+} d S-\int_{\Gamma_{e}} z{\frac{\partial w_{\sigma}^{2}}{\partial \nu}}^{2} d S \\
& \leq\|\psi\|_{H^{1 / 2}(\partial D)}\left\|\frac{\partial w_{\sigma}^{+}}{\partial \nu}\right\|_{H^{-1 / 2}(\partial D)},
\end{aligned}
$$


where we used the jump and boundary conditions of (3.9). Due to the boundedness of the mapping (see p. 381 in [5]),

$$
H(\operatorname{div}, \Omega \backslash \bar{D}) \rightarrow H^{-1 / 2}(\partial D),\left.\quad \mathbf{v} \mapsto(\nu \cdot \mathbf{v})^{+}\right|_{\partial D},
$$

where $H(\operatorname{div}, \Omega \backslash \bar{D})=\left\{\mathbf{v} \in L^{2}(\Omega \backslash \bar{D})^{n} \mid \nabla \cdot \mathbf{v} \in L^{2}(\Omega \backslash \bar{D})\right\}$, it is true that

$$
\left\|\frac{\partial w_{\sigma}^{+}}{\partial \nu}\right\|_{H^{-1 / 2}(\partial D)} \leq C\left\|\nabla w_{\sigma}\right\|_{L^{2}(\Omega)}
$$

where $C>0$ has nothing to do with $\Gamma_{e}$. Using this once in (3.13), we get

$$
\left\|\nabla w_{\sigma}\right\|_{L^{2}(\Omega)} \leq C\|\psi\|_{H^{1 / 2}(\partial D)},
$$

from which it follows that the only solution of (3.9) corresponding to $\psi=0$ is the zero element of $\left(H^{1}(\Omega \backslash \partial D) \oplus T\right) / \mathbb{R}$. Consequently, diffraction problem (3.9) has a unique solution. Together with (3.15), (3.16) also proves (3.10), which completes the proof.

Corollary 3.6. For $\psi \in H_{0}^{1 / 2}(\partial D)$, the solution of diffraction problem (3.9), $\left(w_{\sigma}, W_{\sigma}\right) \in\left(H^{1}(\Omega \backslash \partial D) \oplus T\right) / \mathbb{R}$, is the unique minimizer of the energy functional

$$
E_{\sigma}(w, W)=\int_{\Omega \backslash \bar{D}}|\nabla w|^{2} d x+\kappa \int_{D}|\nabla w|^{2} d x+\int_{\Gamma_{e}} \frac{1}{z}|W-w|^{2} d S
$$

over the subset

$$
H_{\psi}=\left\{(w, W) \in\left(H^{1}(\Omega \backslash \partial D) \oplus T\right) / \mathbb{R} \mid[w]_{\partial D}=\psi\right\} .
$$

Proof. Let $(w, W) \in H_{\psi}$ be arbitrary and denote the difference $\left(w-w_{\sigma}, W-W_{\sigma}\right)$ by $(v, V)$. In consequence, $(v, V) \in\left(H^{1}(\Omega \backslash \partial D) \oplus T\right) / \mathbb{R}$ with $[v]_{\partial D}=0$ and we may write

$$
\begin{aligned}
E_{\sigma}(w, W)= & E_{\sigma}\left(w_{\sigma}, W_{\sigma}\right)+E_{\sigma}(v, V)+2\left\{\int_{\Omega \backslash \bar{D}} \nabla w_{\sigma} \cdot \nabla v d x+\kappa \int_{D} \nabla w_{\sigma} \cdot \nabla v d x\right. \\
& \left.+\int_{\Gamma_{e}} \frac{1}{z}\left(W_{\sigma}-w_{\sigma}\right)(V-v) d S\right\} .
\end{aligned}
$$

We claim that the mixed terms on the right-hand side of (3.17) vanish.

Indeed, by Green's formula

$$
\begin{aligned}
\int_{\Omega \backslash \bar{D}} \nabla w_{\sigma} \cdot \nabla v d x+\kappa \int_{D} \nabla w_{\sigma} \cdot \nabla v d x & =\int_{\partial \Omega} \frac{\partial w_{\sigma}}{\partial \nu} v d S+\int_{\partial D}\left(\kappa{\frac{\partial w_{\sigma}}{\partial \nu}}^{-}-{\frac{\partial w_{\sigma}}{\partial \nu}}^{+}\right) v d S \\
& =\int_{\Gamma_{e}} \frac{\partial w_{\sigma}}{\partial \nu} v d S
\end{aligned}
$$

due to the jump condition of the normal derivative in (3.9). On the other hand,

$$
\int_{\Gamma_{e}} \frac{1}{z}\left(W_{\sigma}-w_{\sigma}\right)(V-v) d S=\int_{\Gamma_{e}} \frac{\partial w_{\sigma}}{\partial \nu}(V-v) d S=-\int_{\Gamma_{e}} \frac{\partial w_{\sigma}}{\partial \nu} v d S
$$

which, together with $(3.17),(3.18)$, and the positivity of $E_{\sigma}$, implies that

$$
E_{\sigma}(w, W) \geq E_{\sigma}\left(w_{\sigma}, W_{\sigma}\right) .
$$


Since $E_{\sigma}(v, V)=0$ implicates that $v$, with $[v]_{\partial D}=0$, is constant on $\Omega$ and that $V=\left.v\right|_{\Gamma_{e}}$, the inequality in (3.20) is strict for $(w, W) \neq\left(w_{\sigma}, W_{\sigma}\right)$ in $H_{\psi}$, which completes the proof.

Lemma 3.7. The operator $F: H_{0}^{1 / 2}(\partial D) \rightarrow H_{0}^{-1 / 2}(\partial D)$ is well defined, continuous, self-adjoint, and bijective, with a continuous inverse operator $F^{-1}: H_{0}^{-1 / 2}(\partial D) \rightarrow$ $H_{0}^{1 / 2}(\partial D)$. In addition, the operator $\operatorname{sgn}(1-\kappa) F: H_{0}^{1 / 2}(\partial D) \rightarrow H_{0}^{-1 / 2}(\partial D)$ is positive. Furthermore, there exist constants $C^{+}, C^{-}>0$, independent of the geometry of $\Gamma_{e}$ as a subset of $\partial \Omega$, such that

$$
\|F\| \leq C^{+}, \quad\left\|F^{-1}\right\| \leq C^{-} .
$$

Proof. For $\psi \in H_{0}^{1 / 2}(\partial D)$, let $\left(w_{\sigma}, W_{\sigma}\right),\left(w_{\mathbf{1}}, W_{\mathbf{1}}\right) \in\left(H^{1}(\Omega \backslash \partial D) \oplus T\right) / \mathbb{R}$ be the solutions of (3.9) corresponding to the conductivities $\sigma$ and $\mathbf{1}$, respectively. First of all, according to the divergence theorem,

$$
\int_{\partial D} \frac{\partial\left(w_{\sigma}-w_{\mathbf{1}}\right)^{+}}{\partial \nu} d S=\int_{\partial \Omega} \frac{\partial\left(w_{\sigma}-w_{\mathbf{1}}\right)}{\partial \nu} d S=0
$$

from which it follows that $\left.{\frac{\partial\left(w_{\sigma}-w_{1}\right)}{\partial \nu}}^{+}\right|_{\partial D} \in H_{0}^{-1 / 2}(\partial D)$. Together with Lemma 3.5, this proves that $F$ is well defined and continuous and that the first part of (3.21) holds.

Next we want to establish the self-adjointness. To this end, for $\psi_{1}, \psi_{2} \in H_{0}^{1 / 2}(\partial D)$ let $\left(w_{1}, W_{1}\right),\left(w_{2}, W_{2}\right)$ be the corresponding solutions of diffraction problem (3.9) with the conductivity $\sigma$. By using Green's formula and the boundary conditions of (3.9), we may write

$$
\begin{aligned}
& \int_{\partial D}{\frac{\partial w_{1}}{\partial \nu}}^{+} \psi_{2} d S=\int_{\partial D}{\frac{\partial w_{1}}{\partial \nu}}^{+} w_{2}^{+} d S-\int_{\partial D} \kappa{\frac{\partial w_{1}}{\partial \nu}}^{-} w_{2}^{-} d S \\
& =\int_{\partial \Omega}\left(\frac{\partial w_{1}}{\partial \nu} w_{2}-w_{1} \frac{\partial w_{2}}{\partial \nu}\right) d S+\int_{\partial D}\left(w_{1}^{+}{\frac{\partial w_{2}}{\partial \nu}}^{+}-\kappa w_{1}^{-}{\frac{\partial w_{2}}{\partial \nu}}^{-}\right) d S \\
& =\int_{\Gamma_{e}} \frac{\partial w_{1}}{\partial \nu}\left(W_{2}-z \frac{\partial w_{2}}{\partial \nu}\right) d S-\int_{\Gamma_{e}} \frac{\partial w_{2}}{\partial \nu}\left(W_{1}-z \frac{\partial w_{1}}{\partial \nu}\right) d S \\
& +\int_{\partial D}\left(w_{1}^{+}-w_{1}^{-}\right){\frac{\partial w_{2}}{\partial \nu}}^{+} d S=\int_{\partial D} \psi_{1}{\frac{\partial w_{2}}{\partial \nu}}^{+} d S .
\end{aligned}
$$

Since this holds also for $\mathbf{1}$ as conductivity, we actually have

$$
\left\langle F \psi_{1}, \psi_{2}\right\rangle_{L^{2}(\partial D)}=\left\langle F \psi_{2}, \psi_{1}\right\rangle_{L^{2}(\partial D)}
$$

i.e., $F$ is self-adjoint.

Next we prove the positiveness of $\operatorname{sgn}(1-\kappa) F: H_{0}^{1 / 2}(\partial D) \rightarrow H_{0}^{-1 / 2}(\partial D)$. For $\psi \in$ $H_{0}^{1 / 2}(\partial D), \psi \neq 0$, let $\left(w_{\sigma}, W_{\sigma}\right)$ and $\left(w_{\mathbf{1}}, W_{\mathbf{1}}\right)$ be the solutions of (3.9) corresponding to the conductivities $\sigma$ and $\mathbf{1}$, respectively. By careful use of Green's formula and the jump conditions of (3.9), we deduce

$$
\begin{aligned}
-\int_{\partial D}{\frac{\partial w_{\sigma}^{+}}{\partial \nu} \psi d S}^{+} & =\kappa \int_{\partial D} \frac{\partial w_{\sigma}^{-}}{\partial \nu} w_{\sigma}^{-} d S-\int_{\partial D} \frac{\partial w_{\sigma}^{+}}{\partial \nu} w_{\sigma}^{+} d S \\
& =\int_{\Omega \backslash \bar{D}}\left|\nabla w_{\sigma}\right|^{2} d x+\kappa \int_{D}\left|\nabla w_{\sigma}\right|^{2} d x-\int_{\Gamma_{e}} \frac{\partial w_{\sigma}}{\partial \nu} w_{\sigma} d S \\
& =E_{\sigma}\left(w_{\sigma}, W_{\sigma}\right)
\end{aligned}
$$


where the last equality follows from a slight modification of (3.19) in the proof of Corollary 3.6. Since similar reasoning also applies for $\left(w_{\mathbf{1}}, W_{\mathbf{1}}\right)$, we have altogether

$$
\langle F \psi, \psi\rangle_{L^{2}(\partial D)}=E_{\mathbf{1}}\left(w_{\mathbf{1}}, W_{\mathbf{1}}\right)-E_{\sigma}\left(w_{\sigma}, W_{\sigma}\right) .
$$

Assume first that $\kappa>1$. Then, according to Corollary 3.6, it holds that

$$
E_{\mathbf{1}}\left(w_{\mathbf{1}}, W_{\mathbf{1}}\right)<E_{\mathbf{1}}\left(w_{\sigma}, W_{\sigma}\right) \leq E_{\sigma}\left(w_{\sigma}, W_{\sigma}\right) .
$$

Similarly, for $\kappa<1$ we have

$$
E_{\sigma}\left(w_{\sigma}, W_{\sigma}\right)<E_{\sigma}\left(w_{1}, W_{1}\right) \leq E_{\mathbf{1}}\left(w_{1}, W_{1}\right) .
$$

Together with (3.22), these estimates prove the claim.

Then it is time to concentrate on the invertibility of $F$, beginning with the injectivity. Let $\psi \in H_{0}^{1 / 2}(\partial D)$ be such that $F \psi=0$, meaning that the restricted difference $\left(\left.\left(w_{\sigma}-w_{\mathbf{1}}\right)\right|_{\Omega \backslash \bar{D}}, W_{\sigma}-W_{\mathbf{1}}\right) \in\left(H^{1}(\Omega \backslash \bar{D}) \oplus T\right) / \mathbb{R}$ of the solutions to (3.9) satisfies boundary value problem (3.4) with $\phi=0$. Thus, it follows from the unique solvability of (3.4) (see Theorem 2.3) that $w_{\sigma}=w_{1}+c, c \in \mathbb{R}$, on $\Omega \backslash \bar{D}$, and as a consequence $w_{\sigma}^{-}=w_{\sigma}^{+}-\psi=w_{1}^{+}-\psi+c=w_{1}^{-}+c$ on $\partial D$. Hence, from the unique solvability of the Dirichlet problem

$$
\Delta w=0 \quad \text { in } D, \quad w=w_{1}^{-} \quad \text { on } \partial D
$$

it follows that $w_{\sigma}=w_{1}+c$ also in $D$. Combining these with the jump conditions of the normal derivatives in (3.9), on $\partial D$ we have

$$
{\frac{\partial w_{\sigma}}{\partial \nu}}^{-}={\frac{\partial w_{\mathbf{1}}}{\partial \nu}}^{-}={\frac{\partial w_{\mathbf{1}}}{\partial \nu}}^{+}={\frac{\partial w_{\sigma}}{\partial \nu}}^{+}=\kappa{\frac{\partial w_{\sigma}}{\partial \nu}}^{-}
$$

i.e, all these normal derivatives must vanish. In consequence, $\left(w_{\sigma}, W_{\sigma}\right)$ satisfies (3.4) with $\phi=0$ in $\Omega \backslash \bar{D}$ and, in addition, $w_{\sigma}$ satisfies Neumann problem with zero input current in $D$, meaning that $\left.w_{\sigma}\right|_{\Omega \backslash \bar{D}}$ and $\left.w_{\sigma}\right|_{D}$ equal constants. Hence, $\psi=w_{\sigma}^{+}-w_{\sigma}^{-} \in$ $H_{0}^{1 / 2}(\partial D)$ equals a constant which must be zero due to the normalization condition. Thus, $F: H_{0}^{1 / 2}(\partial D) \rightarrow H_{0}^{-1 / 2}(\partial D)$ is injective.

Next we move on to prove the surjectivity of $F$. For arbitrarily chosen $\phi \in$ $H_{0}^{-1 / 2}(\partial D)$ we aim to construct $\psi \in H_{0}^{1 / 2}(\partial D)$ such that $F \psi=\phi$. First, we define an auxiliary pair $(v, V) \in\left(H^{1}(\Omega \backslash \bar{D}) \oplus T\right) / \mathbb{R}$ as the unique solution of (3.4) with the input current $\phi$ on $\partial D$, and we continue $v$ to $D$ as the unique $H^{1}$-solution of the Dirichlet problem

$$
\Delta v=0 \quad \text { in } D, \quad v^{-}=v^{+} \quad \text { on } \partial D
$$

Hence, $(v, V) \in\left(H^{1}(\Omega \backslash \partial D) \oplus T\right) / \mathbb{R}$ with $[v]_{\partial D}=0$. Further, we define $\varphi=$ $\phi-\left.\kappa \frac{\partial v}{\partial \nu}^{-}\right|_{\partial D}$ and note that $\varphi \in H_{0}^{-1 / 2}(\partial D)$ since $\int_{\partial D} \frac{\partial v}{\partial \nu}^{-} d S=0$ due to the divergence theorem.

The next step is to define the diffraction solution corresponding to the unit conductivity. In the exterior domain $\Omega \backslash \bar{D}$ we choose $\left(w_{\mathbf{1}}, W_{\mathbf{1}}\right) \in\left(H^{1}(\Omega \backslash \bar{D}) \oplus T\right) / \mathbb{R}$ to be the unique solution of (3.4) with $\phi=\frac{1}{\kappa-1} \varphi$, whereas in the inner domain $D$ we define $w_{1}$ to be the unique $H^{1}$-solution of the Neumann problem

$$
\Delta w_{1}=0 \quad \text { in } D, \quad \frac{\partial w_{\mathbf{1}}^{-}}{\partial \nu}=\frac{1}{\kappa-1} \varphi \quad \text { on } \partial D, \quad \int_{\partial D} w_{\mathbf{1}}^{-} d S=\int_{\partial D} w_{1}^{+} d S
$$


Clearly, $\left(w_{\mathbf{1}}, W_{\mathbf{1}}\right) \in\left(H^{1}(\Omega \backslash \partial D) \oplus T\right) / \mathbb{R}$. As mentioned above, $\left(w_{\mathbf{1}}, W_{\mathbf{1}}\right)$ plays here the role of the solution to diffraction problem (3.9) with conductivity $\mathbf{1}$ and, hence, we set $\psi=\left[w_{\mathbf{1}}\right]_{\partial D}$, which belongs to $H_{0}^{1 / 2}(\partial D)$ because of the normalization condition in (3.24). It is a straightforward task to check that the pairs $\left(w_{\mathbf{1}}, W_{\mathbf{1}}\right),\left(w_{\sigma}, W_{\sigma}\right)=$ $\left(w_{\mathbf{1}}+v, W_{\mathbf{1}}+V\right) \in\left(H^{1}(\Omega \backslash \partial D) \oplus T\right) / \mathbb{R}$ satisfy diffraction problem (3.9) for the conductivities 1 and $\sigma$, respectively. Moreover, it holds that

$$
F \psi=\left.{\frac{\partial\left(w_{\sigma}-w_{\mathbf{1}}\right)}{\partial \nu}}^{+}\right|_{\partial D}=\left.\frac{\partial v}{\partial \nu}^{+}\right|_{\partial D}=\phi,
$$

which proves that $F: H_{0}^{1 / 2}(\partial D) \rightarrow H_{0}^{-1 / 2}(\partial D)$ is surjective.

It is a consequence of the open mapping theorem that the inverse of the bijective bounded linear operator $F$ is also bounded. Moreover, by walking the above constructional proof of the surjectivity in the opposite direction and using the continuous dependence on the boundary data of (3.4), (3.23), and (3.24), one easily sees that $F^{-1}: H_{0}^{-1 / 2}(\partial D) \rightarrow H_{0}^{1 / 2}(\partial D)$ is, actually, uniformly bounded with respect to the choice of the electrode configuration, i.e., with respect to the geometry of $\Gamma_{e}$ as a subset of $\partial \Omega$. This completes the proof.

Now we have gathered enough weaponry to prove the factorization of $R_{\sigma}-R_{\mathbf{1}}$.

Proof of Theorem 3.3. For a fixed electrode current $I \in T_{0}$ denote by $\left(u_{\sigma}, U_{\sigma}\right)$, $\left(u_{1}, U_{1}\right) \in H^{1}(\Omega) \oplus T_{0}$ the solutions of the complete electrode forward problem, given in (3.1), with conductivities $\sigma$ and $\mathbf{1}$, respectively. Since $u_{\sigma}-u_{\mathbf{1}}$ is harmonic in $\Omega \backslash \bar{D}$, it follows easily by using the divergence theorem and the complete electrode boundary conditions that

$$
\int_{\partial D} \frac{\partial\left(u_{\sigma}-u_{\mathbf{1}}\right)^{+}}{\partial \nu} d S=\int_{\partial \Omega} \frac{\partial\left(u_{\sigma}-u_{\mathbf{1}}\right)}{\partial \nu} d S=0
$$

Thus, $\left(\left.\left(u_{\sigma}-u_{\mathbf{1}}\right)\right|_{\Omega \backslash \bar{D}}, U_{\sigma}-U_{\mathbf{1}}\right)$ solves (3.4) for $\phi=\left.{\frac{\partial\left(u_{\sigma}-u_{1}\right)}{\partial \nu}}^{+}\right|_{\partial D}$ and, in particular,

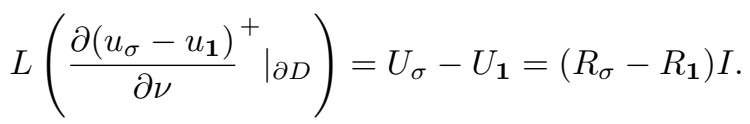

By introducing the operator $G_{\sigma}:\left.I \mapsto{\frac{\partial u_{\sigma}}{\partial \nu}}^{+}\right|_{\partial D}$ and setting $G=G_{\sigma}-G_{1}$, we have so far derived the factorization

$$
R_{\sigma}-R_{1}=L G
$$

Note that $G$ is a well-defined bounded operator from $T_{0}$ to $H_{0}^{-1 / 2}(\partial D)$ due to Theorem 2.3 and (3.14).

The next task is to calculate the dual operator $G_{\sigma}^{\prime}: H_{0}^{1 / 2}(\partial D) \rightarrow T_{0}$ of $G_{\sigma}$. To this end, consider $\left(w_{\sigma}, W_{\sigma}\right) \in H^{1}(\Omega \backslash \partial D) \oplus T_{0}$ the solution of diffraction problem (3.9), with a fixed ground level of the potential, corresponding to $\psi \in H_{0}^{1 / 2}(\partial D)$. With the help of the jump conditions $\left[u_{\sigma}\right]_{\partial D}=\left[\sigma \frac{\partial u_{\sigma}}{\partial \nu}\right]_{\partial D}=0$ (cf. [8]), $\left[\sigma \frac{\partial w_{\sigma}}{\partial \nu}\right]_{\partial D}=0$, Green's formula, and the boundary conditions on $u_{\sigma}$ and $w_{\sigma}$, we deduce

$$
\begin{aligned}
\left\langle G_{\sigma} I, \psi\right\rangle_{L^{2}(\partial D)} & =\int_{\partial D} \frac{\partial u_{\sigma}^{+}}{\partial \nu} w_{\sigma}^{+} d S-\int_{\partial D} \kappa{\frac{\partial u_{\sigma}^{-}}{\partial \nu}}^{-} w_{\sigma}^{-} d S \\
& =\int_{\partial D}\left(\frac{\partial w_{\sigma}^{+}}{\partial \nu} u_{\sigma}^{+}-\kappa{\frac{\partial w_{\sigma}}{\partial \nu}}^{-} u_{\sigma}^{-}\right) d S+\int_{\partial \Omega}\left(\frac{\partial u_{\sigma}}{\partial \nu} w_{\sigma}-\frac{\partial w_{\sigma}}{\partial \nu} u_{\sigma}\right) d S
\end{aligned}
$$




$$
\begin{aligned}
& =\int_{\Gamma_{e}} \frac{\partial u_{\sigma}}{\partial \nu} w_{\sigma} d S+\int_{\Gamma_{e}} \frac{\partial w_{\sigma}}{\partial \nu}\left(U_{\sigma}-u_{\sigma}\right) d S=\int_{\Gamma_{e}} \frac{\partial u_{\sigma}}{\partial \nu}\left(w_{\sigma}+z \frac{\partial w_{\sigma}}{\partial \nu}\right) d S \\
& =\int_{\Gamma_{e}}\left(\frac{\partial u_{\sigma}}{\partial \nu}-I\right) W_{\sigma} d S+\int_{\Gamma_{e}} I W_{\sigma} d S=\left\langle I, W_{\sigma}\right\rangle_{L^{2}(\partial \Omega)}
\end{aligned}
$$

which shows that $G_{\sigma}^{\prime} \psi=W_{\sigma}$. Hence, with $\left(w_{\mathbf{1}}, W_{\mathbf{1}}\right) \in H^{1}(\Omega \backslash \partial D) \oplus T_{0}$ the solution of diffraction problem (3.9) corresponding to $\psi$ and the unit conductivity, we have

$$
G^{\prime} \psi=W_{\sigma}-W_{1}
$$

The restriction $\left(\left.\left(w_{\sigma}-w_{\mathbf{1}}\right)\right|_{\Omega \backslash \bar{D}}, W_{\sigma}-W_{\mathbf{1}}\right) \in H^{1}(\Omega \backslash \bar{D}) \oplus T_{0}$ solves $(3.4)$ for $\phi=\left.{\frac{\partial\left(w_{\sigma}-w_{1}\right)}{\partial \nu}}^{+}\right|_{\partial D} \in H_{0}^{-1 / 2}(\partial D)$, which means that

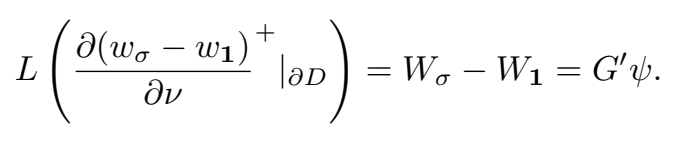

Due to the way $F: H_{0}^{1 / 2}(\partial D) \rightarrow H_{0}^{-1 / 2}(\partial D)$ is defined and since $\psi \in H_{0}^{1 / 2}(\partial D)$ was chosen arbitrarily, relation (3.26) is equivalent to $L F=G^{\prime}$. Taking the transpose of this and plugging it into (3.25), we thus obtain

$$
R_{\sigma}-R_{1}=L F^{\prime} L^{\prime}=L F L^{\prime}
$$

which is what we set out to prove.

3.2. Some further properties of $\boldsymbol{F}, \boldsymbol{L}$, and $\boldsymbol{L}^{\prime}$. We define a new boundary operator by

$$
\left|R_{\sigma}-R_{1}\right|=\operatorname{sgn}(1-\kappa)\left(R_{\sigma}-R_{\mathbf{1}}\right) .
$$

Due to the way we have defined our conductivity in (3.3), it follows trivially from Lemma 3.1 and Corollary 3.2 that $\left|R_{\sigma}-R_{\mathbf{1}}\right|: T_{0} \rightarrow T_{0}$ is self-adjoint and strictly positive. Denoting the operator $\operatorname{sgn}(1-\kappa) F: H_{0}^{1 / 2}(\partial D) \rightarrow H_{0}^{-1 / 2}(\partial D)$ by $|F|$, it follows from Theorem 3.3 that this new boundary operator can be factorized as $\left|R_{\sigma}-R_{\mathbf{1}}\right|=L|F| L^{\prime}$. In the next subsection we will use the operator $\left|R_{\sigma}-R_{\mathbf{1}}\right|$ to characterize the inclusion $D$. However, to be successful in this task, we must devote the ongoing subsection to further investigations of $|F|, L$, and $L^{\prime}$.

LEMmA 3.8. The operator $|F|: H_{0}^{1 / 2}(\partial D) \rightarrow H_{0}^{-1 / 2}(\partial D)$ can be given as $|F|=$ $F^{1 / 2}\left(F^{1 / 2}\right)^{\prime}$, where $F^{1 / 2}: L_{0}^{2}(\partial D) \rightarrow H_{0}^{-1 / 2}(\partial D)$ and $\left(F^{1 / 2}\right)^{\prime}: H_{0}^{1 / 2}(\partial D) \rightarrow L_{0}^{2}(\partial D)$ are bounded, bijective, and dual to each other. Further, it holds that

$$
\left\|F^{1 / 2}\right\| \leq C^{+} \sqrt{C^{-}}, \quad\left\|F^{-1 / 2}\right\| \leq \sqrt{C^{-}}
$$

where $C^{+}, C^{-}>0$ are the constants introduced in Lemma 3.7 .

Proof. Since $H_{0}^{1 / 2}(\partial D) \hookrightarrow L_{0}^{2}(\partial D) \hookrightarrow H_{0}^{-1 / 2}(\partial D)$ is a Gelfand triple and since $|F|^{-1}: H_{0}^{-1 / 2}(\partial D) \rightarrow H_{0}^{1 / 2}(\partial D)$ is isomorphic, self-adjoint, and positive, it follows from material in [3] that there exists a factorization

$$
|F|^{-1}=\left(F^{-1 / 2}\right)^{\prime} F^{-1 / 2},
$$


where $F^{-1 / 2}: H_{0}^{-1 / 2}(\partial D) \rightarrow L_{0}^{2}(\partial D)$ and $\left(F^{-1 / 2}\right)^{\prime}: L_{0}^{2}(\partial D) \rightarrow H_{0}^{1 / 2}(\partial D)$ are bounded, bijective, and dual to each other, with

$$
\left\|F^{-1 / 2}\right\|=\left\|\left(F^{-1 / 2}\right)^{\prime}\right\| \leq\left\|F^{-1}\right\|^{1 / 2} \leq \sqrt{C^{-}} .
$$

Further, for $\eta \in L_{0}^{2}(\partial D)$ we may estimate

$$
\left\|F^{1 / 2} \eta\right\|_{H^{-1 / 2}(\partial D)}=\left\||| F \mid\left(F^{-1 / 2}\right)^{\prime} \eta\right\|_{H^{-1 / 2}(\partial \Omega)} \leq C^{+} \sqrt{C^{-}}\|\eta\|_{L^{2}(\partial D)},
$$

where we used Lemma 3.7 and (3.28).

In what follows we denote by $\mathcal{N}(L)^{\perp} \subset H_{0}^{-1 / 2}(\partial D)$ the orthogonal complement of $\mathcal{N}(L) \subset H_{0}^{-1 / 2}(\partial D)$ with respect to the inner product of the Hilbert space $H^{-1 / 2}(\partial D)$. Let $Q: \mathcal{R}\left(F L^{\prime}\right) \rightarrow \mathcal{N}(L)^{\perp}$ be an orthogonal projection; i.e., for $\phi \in \mathcal{R}\left(F L^{\prime}\right) \subset H_{0}^{-1 / 2}(\partial D)$,

$$
Q \phi=\phi_{\perp} \in \mathcal{N}(L)^{\perp} \quad \text { with } L \phi_{\perp}=L \phi .
$$

Note that $Q$ is well defined due to the projection theorem [6]. In addition, we claim that $Q$ is a bijection.

Corollary 3.9. The orthogonal projection $Q: \mathcal{R}\left(F L^{\prime}\right) \rightarrow \mathcal{N}(L)^{\perp}$ defined by (3.29) is bijective with the norm estimates

$$
\|Q\| \leq 1, \quad\left\|Q^{-1}\right\| \leq\left(C^{+} C^{-}\right)^{2}
$$

where $C^{+}, C^{-}>0$ are the constants introduced in Lemma 3.7.

Proof. To begin with, note that the left-hand inequality of (3.30) is obvious. In order to obtain the right-hand inequality, let $\phi \in \mathcal{R}\left(F L^{\prime}\right)=\mathcal{R}\left(|F| L^{\prime}\right)$ and $\phi_{\perp}=Q \phi \in$ $\mathcal{N}(L)^{\perp}$, and note that $\phi-\phi_{\perp} \in \mathcal{N}(L)$. In consequence, we may write

$$
\begin{aligned}
\left\|\phi_{\perp}\right\|_{H^{-1 / 2}(\partial D)} & \geq \sup _{\|\psi\|_{H^{1 / 2}}=1, \psi \in \mathcal{R}\left(L^{\prime}\right)}\left\langle\phi_{\perp}, \psi\right\rangle_{L^{2}(\partial D)} \\
& =\sup _{\|\psi\|_{H^{1 / 2}}=1, \psi \in \mathcal{R}\left(L^{\prime}\right)}\langle\phi, \psi\rangle_{L^{2}(\partial D)} .
\end{aligned}
$$

Further, since $|F|^{-1} \phi \in \mathcal{R}\left(L^{\prime}\right)$, we have

$$
\left\|\phi_{\perp}\right\|_{H^{-1 / 2}(\partial D)} \geq \frac{1}{\left\||F|^{-1} \phi\right\|_{H^{1 / 2}(\partial D)}}\left\langle\phi,|F|^{-1} \phi\right\rangle_{L^{2}(\partial D)}=\frac{\left\|F^{-1 / 2} \phi\right\|_{L^{2}(\partial D)}^{2}}{\left\||F|^{-1} \phi\right\|_{H^{1 / 2}(\partial D)}},
$$

and so we finally obtain

$$
\left\|\phi_{\perp}\right\|_{H^{-1 / 2}(\partial D)} \geq \frac{\|\phi\|_{H^{-1 / 2}(\partial D)}}{\left\|F^{1 / 2}\right\|^{2}\left\||F|^{-1}\right\|} \geq \frac{1}{\left(C^{+} C^{-}\right)^{2}}\|\phi\|_{H^{-1 / 2}(\partial D)}
$$

by Lemmas 3.7 and 3.8 .

According to Lemmas 3.4 and 3.7, $L: H_{0}^{-1 / 2}(\partial D) \rightarrow T_{0}$ is surjective, $L^{\prime}$ : $T_{0} \rightarrow H_{0}^{1 / 2}(\partial D)$ is injective, and $F: H_{0}^{1 / 2}(\partial D) \rightarrow H_{0}^{-1 / 2}(\partial D)$ is bijective. Thus, $\operatorname{dim}\left(\mathcal{N}(L)^{\perp}\right)=\operatorname{dim}\left(\mathcal{R}\left(F L^{\prime}\right)\right)=\operatorname{dim}\left(T_{0}\right)<\infty$, and so the bijectivity of $Q: \mathcal{R}\left(F L^{\prime}\right) \rightarrow$ $\mathcal{N}(L)^{\perp}$ follows from its injectivity that is guaranteed by (3.31), which provides also the needed norm estimate for $Q^{-1}$. 
To end this subsection, we make a few comments about the inverse operators of $L$ and $L^{\prime}$ defined in (3.5) and (3.8), respectively. Since $L: H_{0}^{-1 / 2}(\partial D) \rightarrow T_{0}$ is noninjective and $L^{\prime}: T_{0} \rightarrow H_{0}^{1 / 2}(\partial D)$ is nonsurjective, they do not have inverse operators as such. However, the restrictions $L: \mathcal{R}\left(F L^{\prime}\right) \rightarrow T_{0}$ and $L^{\prime}: T_{0} \rightarrow \mathcal{R}\left(L^{\prime}\right)$ do have bounded inverses due to the bijectivity of $R_{\sigma}-R_{1}=L F L^{\prime}: T_{0} \rightarrow T_{0}$ and finite-dimensionality of $T_{0}$. In what follows, we will denote by $L^{-1}$ and $\left(L^{\prime}\right)^{-1}$ the inverses of these restrictions, i.e.,

$$
L^{-1}: T_{0} \rightarrow \mathcal{R}\left(F L^{\prime}\right), \quad\left(L^{\prime}\right)^{-1}: \mathcal{R}\left(L^{\prime}\right) \rightarrow T_{0},
$$

with $L L^{-1}=$ id, $\left.L^{-1} L\right|_{\mathcal{R}\left(F L^{\prime}\right)}=$ id and $L^{\prime}\left(L^{\prime}\right)^{-1}=$ id, $\left(L^{\prime}\right)^{-1} L^{\prime}=$ id. With this notation, we can factorize $\left|R_{\sigma}-R_{\mathbf{1}}\right|^{-1}: T_{0} \rightarrow T_{0}$, which exists according to Lemma 3.2 , as

$$
\left|R_{\sigma}-R_{\mathbf{1}}\right|^{-1}=\left(L^{\prime}\right)^{-1}|F|^{-1} L^{-1}=\left(L^{\prime}\right)^{-1}\left(F^{-1 / 2}\right)^{\prime} F^{-1 / 2} L^{-1},
$$

due to Theorem 3.3 and Lemma 3.8.

3.3. Characterizing the inclusion. Before we can formulate and prove the main result of this section, we need to introduce some new concepts. Let $\left\{\mathcal{T}_{M}\right\}$ be a sequence of electrode configurations, meaning that

$$
\mathcal{T}_{M}=\left\{e_{1}^{M}, \ldots, e_{M}^{M} \subset \partial \Omega \mid e_{l}^{M} \cap e_{m}^{M}=\emptyset \text { if } l \neq m\right\}, \quad \Gamma_{M}=\cup_{m=1}^{M} e_{m}^{M},
$$

for each $1 \leq M<\infty$, satisfying the following conditions: $d\left(e_{m}^{M}\right) \leq \beta_{M}$ for all $1 \leq$ $m \leq M$,

$$
\left|\partial \Omega \backslash \Gamma_{M}\right|, \beta_{M} \rightarrow 0 \quad \text { when } M \rightarrow \infty,
$$

where $d\left(e_{m}^{M}\right)$ is the diameter of $e_{m}^{M}$, i.e., $d\left(e_{m}^{M}\right)=\sup _{x, y \in e_{m}^{M}}|x-y|$. The subspaces $T^{M}$ and $T_{0}^{M}$, corresponding to the electrode configuration $\mathcal{T}_{M}$, are defined in accordance with (2.5) and (2.6), respectively, and the associated orthogonal projections $P_{1}^{M}$ : $L_{0}^{2}(\partial \Omega) \rightarrow L_{0}^{2}\left(\Gamma_{M}\right), P_{2}^{M}: L_{0}^{2}\left(\Gamma_{M}\right) \rightarrow T_{0}^{M}$, and $P^{M}: L_{0}^{2}(\partial \Omega) \rightarrow T_{0}^{M}$ are given by obvious modifications of (2.3), (2.9), and (2.26). We also will use a similar index notation for other operators depending on the used electrode configuration.

Let $y \in \Omega$ be a parameter and $\hat{\alpha} \in \mathbb{R}^{n}$ a unit vector, and consider the solution $\Phi_{y}$ of the following homogenous Neumann problem:

$$
\Delta \Phi(x)=\hat{\alpha} \cdot \nabla \delta(x-y) \quad \text { in } \Omega, \quad \frac{\partial \Phi}{\partial \nu}=0 \quad \text { on } \partial \Omega, \quad \int_{\partial \Omega} \Phi d S=0,
$$

where $\delta$ is the delta functional. Physically $\Phi_{y}$ corresponds to the electromagnetic potential created by a dipole point source at $y$ pointing in the direction $\hat{\alpha}$. It is a well-known fact that (3.34) is uniquely solvable with $\Phi_{y} \in C^{\infty}(\Omega \backslash\{y\})$ and $\Phi_{y}$ singular at $y$.

Assume that (3.33) is valid and let $\left\{\alpha_{M}\right\} \subset \mathbb{R}_{+}$be a sequence of regularization parameters. Consider the minimizing sequence $\left\{I^{M}\right\} \subset L_{0}^{2}(\partial \Omega), I^{M} \in T_{0}^{M}$, of the Tikhonov functionals

$$
\left\|\left|R_{\sigma}^{M}-R_{\mathbf{1}}^{M}\right|^{1 / 2} I-\Phi_{y}\right\|_{L^{2}(\partial \Omega)}^{2}+\alpha_{M}\|I\|_{L^{2}(\partial \Omega)}^{2}, \quad I \in T_{0}^{M}, 1 \leq M<\infty,
$$

where $\left|R_{\sigma}^{M}-R_{1}^{M}\right|^{1 / 2}: T_{0}^{M} \rightarrow T_{0}^{M}$ is the unique, positive, self-adjoint, bijective square root of $\left|R_{\sigma}^{M}-R_{1}^{M}\right|$ defined in (3.27). Since $R_{\sigma}^{M}-R_{1}^{M}$ can be obtained through boundary measurements, so can $\left|R_{\sigma}^{M}-R_{1}^{M}\right|^{1 / 2}$ and, hence, the behavior of the sequence 
$\left\{I^{M}\right\}$ is something that can be observed by noninvasive methods. The following theorem characterizes the inclusion $D$ by the limit behavior of $\left\{I^{M}\right\}$.

THEOREM 3.10. Assume that (3.33) holds and the contact impedance $z$ is smooth. Let $\left\{I^{M}\right\} \subset L_{0}^{2}(\partial \Omega), I^{M} \in T_{0}^{M}$, be the minimizing sequence for the functionals (3.35) and assume that $\left\{\alpha_{M}\right\} \subset \mathbb{R}_{+}$converges to zero but is such that the sequence

$$
\left\{\frac{\inf _{V \in T^{M}}\left\|\Phi_{y}-V\right\|_{L^{2}(\partial \Omega)}^{2}}{\alpha_{M}}\right\}
$$

is bounded. Then $y \in D$ if and only if the sequence $\left\{I^{M}\right\}$ is bounded in $L_{0}^{2}(\partial \Omega)$.

In real life one is, naturally, not able to construct a sequence of electrode configurations with the properties given in (3.33). However, when conducting measurements with a fixed setting of electrodes that are relatively small and cover a large portion of the object boundary, Theorem 3.10 gives a reason to believe that the electrode currents needed for minimizing functional (3.35), with a fixed small $\alpha>0$, are larger when $y \in \Omega \backslash D$ than when $y \in D$. This observation leads to a possibility of numerical implementation that will be considered in forthcoming articles.

The following simple lemma shows that the conditions of Theorem 3.10 are reasonable. One could also quite easily derive a quantitative estimate to suggest an a priori choice of regularization parameters $\alpha_{M}$ in Theorem (3.10) but for simplicity we content ourselves with a mere convergence result.

Lemma 3.11. Let $f \in C^{\infty}(\partial \Omega)$ and assume that $\left\{\mathcal{T}_{M}\right\}$ satisfies (3.33). Then it holds that

$$
\inf _{V \in T^{M}}\|f-V\|_{L^{2}(\partial \Omega)} \rightarrow 0
$$

when $M$ goes to infinity.

Proof. The claim is a straightforward consequence of the good behavior of $\left\{\mathcal{T}_{M}\right\}$ given by (3.33).

The rest of this section is devoted to the proof of Theorem 3.10. Let $L^{M}$ be the operator defined in (3.5) corresponding to the electrode configuration $\mathcal{T}_{M}$. We define the associated limit operator $\tilde{L}: H_{0}^{-1 / 2}(\partial D) \rightarrow H_{0}^{1 / 2}(\partial \Omega)$ by

$$
\tilde{L} \phi=\left.v\right|_{\partial \Omega}, \quad \phi \in H_{0}^{-1 / 2}(\partial D),
$$

where $v \in H_{0, \partial \Omega}^{1}(\Omega \backslash \bar{D})$ is the unique solution of the boundary value problem

$$
\Delta v=0 \quad \text { in } \Omega \backslash \bar{D}, \quad \frac{\partial v}{\partial \nu}^{+}=\phi \quad \text { on } \partial D, \quad \frac{\partial v}{\partial \nu}=0 \quad \text { on } \partial \Omega .
$$

The adjoint of $\tilde{L}$ is $\tilde{L}^{\prime}: H_{0}^{-1 / 2}(\partial \Omega) \rightarrow H_{0}^{1 / 2}(\partial D)[3]$,

$$
\tilde{L}^{\prime} \phi^{\prime}=\left.v^{\prime}\right|_{\partial D}, \quad \phi^{\prime} \in H_{0}^{-1 / 2}(\partial \Omega)
$$

where $v^{\prime} \in H_{0, \partial D}^{1}(\Omega \backslash \bar{D})$ is the unique solution of the boundary value problem

$$
\Delta v^{\prime}=0 \quad \text { in } \Omega \backslash \bar{D}, \quad \frac{\partial v^{\prime}}{\partial \nu}=-\phi^{\prime} \quad \text { on } \partial \Omega, \quad \frac{\partial v^{+}}{\partial \nu}=0 \quad \text { on } \partial D .
$$

The first step of our proof is to characterize the inclusion $D$ by the operator sequence $\left\{L_{M}\right\}$ with the help of known mapping properties of $\tilde{L}$ and $\tilde{L}^{\prime}$. 
Assume that $\left\{\alpha_{M}\right\}$ is a sequence of positive regularization parameters that converges to zero and consider the following Tikhonov functionals:

$$
\left\|L^{M} \phi-\Phi_{y}\right\|_{L^{2}(\partial \Omega)}^{2}+\alpha_{M}\|\phi\|_{H^{-1 / 2}(\partial D)}^{2}, \quad 1 \leq M \leq \infty .
$$

Since $L^{M}: H_{0}^{-1 / 2}(\partial D) \rightarrow T_{0}^{M} \subset L_{0}^{2}(\partial \Omega)$ is continuous for every $M \in \mathbb{N}$, it is well known that each of these functionals has a unique minimizer $\phi^{M} \in H_{0}^{-1 / 2}(\partial D)$. We intend to show that, for a correctly chosen sequence of regularization parameters $\left\{\alpha_{M}\right\}$, the behavior of the minimizer sequence $\left\{\phi^{M}\right\}$ at infinity determines uniquely whether $y$ belongs to the inclusion $D$ or not. We begin with the case when $y \in \Omega \backslash D$.

Lemma 3.12. Assume that $y \in \Omega \backslash D$, the contact impedance $z$ is smooth, and $\left\{\alpha_{M}\right\} \subset \mathbb{R}_{+}$converges to zero. Let $\left\{\phi^{M}\right\} \subset H_{0}^{-1 / 2}(\partial D)$ be the minimizing sequence for the functionals (3.38). Then it holds that

$$
\left\|\phi^{M}\right\|_{H^{-1 / 2}(\partial D)} \rightarrow \infty
$$

as $M$ goes to infinity.

Proof. First, we will show that $L^{M} \phi^{M}$ converges to $\left.\Phi_{y}\right|_{\partial \Omega}$ as $M$ goes to infinity. Let $\epsilon>0$ be given. Since $\tilde{L}^{\prime}$ defined in (3.37) is clearly injective, we have $\overline{\mathcal{R}(\tilde{L})}=$ $\mathcal{N}\left(\tilde{L}^{\prime}\right)^{\perp}=H_{0}^{1 / 2}(\partial \Omega)$, where the orthogonal complement is taken with respect to the dual pairing between $H_{0}^{-1 / 2}(\partial \Omega)$ and $H_{0}^{1 / 2}(\partial \Omega)$. Hence, $\mathcal{R}(\tilde{L})$ is dense in $H_{0}^{1 / 2}(\partial \Omega)$ and, thus, also in $L_{0}^{2}(\partial \Omega)$. In consequence, we can choose $\phi^{\epsilon} \in H_{0}^{-1 / 2}(\partial D)$ such that

$$
\left\|\tilde{L} \phi^{\epsilon}-\Phi_{y}\right\|_{L^{2}(\partial \Omega)}^{2}<\frac{\epsilon^{2}}{6} .
$$

Note also that $\tilde{L} \phi^{\epsilon} \in C^{\infty}(\partial \Omega) \cap H_{0}^{1 / 2}(\partial \Omega)$ due to the regularity theory of elliptic partial differential equations [10].

Since $\tilde{L} \phi^{\epsilon} \in H_{0}^{1 / 2}(\partial \Omega)$ and $L^{M} \phi^{\epsilon} \in L_{0}^{2}\left(\Gamma_{M}\right)$, by using the projection $P_{1}^{M}$ : $L_{0}^{2}(\partial \Omega) \rightarrow L_{0}^{2}\left(\Gamma_{M}\right)$, defined by $(2.3)$, we can estimate

$$
\begin{aligned}
\left\|\left(\tilde{L}-L^{M}\right) \phi^{\epsilon}\right\|_{L^{2}\left(\Gamma_{M}\right)} & \leq\left\|\tilde{L} \phi^{\epsilon}-P_{1}^{M}\left(\tilde{L} \phi^{\epsilon}\right)\right\|_{L^{2}\left(\Gamma_{M}\right)}+\left\|P_{1}^{M}\left(\tilde{L}-L^{M}\right) \phi^{\epsilon}\right\|_{L^{2}\left(\Gamma_{M}\right)} \\
& \leq C\left\{\frac{\left|\partial \Omega \backslash \bar{\Gamma}_{M}\right|^{1 / 2}}{\left|\Gamma_{M}\right|^{1 / 2}}\left\|\tilde{L} \phi^{\epsilon}\right\|_{L^{2}\left(\partial \Omega \backslash \bar{\Gamma}_{M}\right)}+\left\|\left(\tilde{L}-L^{M}\right) \phi^{\epsilon}\right\| \|_{L^{2}\left(\Gamma_{M}\right) / \mathbb{R}}\right\} \\
& \leq C\left\{\frac{\left|\partial \Omega \backslash \bar{\Gamma}_{M}\right|^{1 / 2}}{\left|\Gamma_{M}\right|^{1 / 2}}\left\|\tilde{L} \phi^{\epsilon}\right\|_{L^{2}\left(\partial \Omega \backslash \bar{\Gamma}_{M}\right)}+\inf _{V \in T^{M}}\left\|\tilde{L} \phi^{\epsilon}-V\right\|_{L^{2}\left(\Gamma_{M}\right)}\right\},
\end{aligned}
$$

where the second-to-last inequality follows from (2.3), by using the Schwarz inequality, and the fact that $P_{1}^{M}\left(\tilde{L}-L^{M}\right) \phi^{\epsilon} \in L_{0}^{2}\left(\Gamma_{M}\right)$, and the last inequality follows from Lemma 2.7 and Theorem 2.11 applied on boundary value problems (3.36) and (3.4). Thus, according to Lemma 3.11 , we can choose $M_{0} \in \mathbb{N}$ in such a way that

$$
\left\|\left(L^{M}-\tilde{L}\right) \phi^{\epsilon}\right\|_{L^{2}(\partial \Omega)}^{2} \leq \frac{C}{\left|\Gamma_{M}\right|} \inf _{V \in T^{M}}\left\|\tilde{L} \phi^{\epsilon}-V\right\|_{L^{2}(\partial \Omega)}^{2}<\frac{\epsilon^{2}}{6},
$$

and, in addition,

$$
\alpha_{M}\left\|\phi^{\epsilon}\right\|_{H^{-1 / 2}(\partial D)}^{2}<\frac{\epsilon^{2}}{3}
$$


for all $M \geq M_{0}$. Consequently, due to estimates (3.39), (3.40), (3.41), and the triangle inequality, for every $M \geq M_{0}$ it holds that

$$
\begin{aligned}
\left\|L^{M} \phi^{M}-\Phi_{y}\right\|_{L^{2}(\partial \Omega)}^{2} & +\alpha_{M}\left\|\phi^{M}\right\|_{H^{-1 / 2}(\partial D)}^{2} \\
& \leq\left\|L^{M} \phi^{\epsilon}-\Phi_{y}\right\|_{L^{2}(\partial \Omega)}^{2}+\alpha_{M}\left\|\phi^{\epsilon}\right\|_{H^{-1 / 2}(\partial D)}^{2}<\epsilon^{2} .
\end{aligned}
$$

In particular, since $\epsilon>0$ was chosen arbitrarily, we have obtained

$$
\left\|L^{M} \phi^{M}-\Phi_{y}\right\|_{L^{2}(\partial \Omega)} \rightarrow 0
$$

when $M$ goes to infinity.

Next, we will use contradiction: Assume that the minimizing sequence $\left\{\phi^{M}\right\}$ is bounded in $H_{0}^{-1 / 2}(\partial D)$. In consequence, it follows from fundamental functional analysis [6] that $\left\{\phi^{M}\right\}$ has a subsequence $\left\{\phi^{M_{k}}\right\}_{k=1}^{\infty}$ that converges weakly to some distribution $\phi^{\prime} \in H_{0}^{-1 / 2}(\partial D)$. Our goal is to show that $\tilde{L} \phi^{\prime}=\left.\Phi_{y}\right|_{\partial \Omega}$, which is a contradiction due to the singularity of $\Phi_{y}$ at $y \in \Omega \backslash D[3]$.

Let $g \in C^{\infty}(\partial \Omega) \cap L_{0}^{2}(\partial \Omega)$ be arbitrary and write it in two parts as $g=P_{1}^{M_{k}} g+$ $\left(I-P_{1}^{M_{k}}\right) g$, where $P_{1}^{M_{k}}$ is defined by (2.3). Then we have

$$
\left\langle L^{M_{k}} \phi^{M_{k}}, g\right\rangle_{L^{2}(\partial \Omega)}=\left\langle L^{M_{k}} \phi^{M_{k}}, P^{M_{k}} g\right\rangle_{L^{2}(\partial \Omega)}+\left\langle L^{M_{k}} \phi^{M_{k}},\left(I-P_{1}^{M_{k}}\right) g\right\rangle_{L^{2}\left(\Gamma_{M_{k}}\right)},
$$

where we used the fact that $L^{M_{k}} \phi^{M_{k}}$ is constant over each $e_{m}^{M_{k}}$ and zero elsewhere, and the way $P^{M_{k}}$ is defined in (2.26). Due to the uniform boundedness of the operators $\left\{L^{M_{k}}\right\} \subset \mathcal{L}\left(H_{0}^{-1 / 2}(\partial D), L_{0}^{2}(\partial \Omega)\right.$ ) (see Lemma 3.4) and of the sequence $\left\{\phi^{M}\right\} \subset H_{0}^{-1 / 2}(\partial D)$, the second term on the right-hand side of (3.42) can be estimated by the Schwarz inequality as follows:

$$
\begin{aligned}
\left|\left\langle L^{M_{k}} \phi^{M_{k}},\left(I-P_{1}^{M_{k}}\right) g\right\rangle_{L^{2}\left(\Gamma_{M_{k}}\right)}\right| & \leq \frac{C}{\left|\Gamma_{M_{k}}\right|}\left\{\int_{\Gamma_{M_{k}}}\left|\int_{\partial \Omega \backslash \bar{\Gamma}_{M_{k}}} g d S\right|^{2} d S\right\}^{1 / 2} \\
& \leq \frac{C}{\left|\Gamma_{M_{k}}\right|^{1 / 2}}\left|\partial \Omega \backslash \Gamma_{M_{k}}\right||| g \|_{\infty} \rightarrow 0,
\end{aligned}
$$

when $k$ goes to infinity due to (3.33). On the other hand, for the first term on the right-hand side of (3.42) we may write

$$
\begin{aligned}
\left\langle L^{M_{k}} \phi^{M_{k}}, P^{M_{k}} g\right\rangle_{L^{2}(\partial \Omega)} & =\left\langle\phi^{M_{k}},\left(L^{M_{k}}\right)^{\prime} P^{M_{k}} g\right\rangle_{L^{2}(\partial D)} \\
& =\left\langle\phi^{M_{k}},\left(\left(L^{M_{k}}\right)^{\prime} P^{M_{k}}-\tilde{L}^{\prime}\right) g\right\rangle_{L^{2}(\partial D)}+\left\langle\phi^{M_{k}}, \tilde{L}^{\prime} g\right\rangle_{L^{2}(\partial D)} .
\end{aligned}
$$

Let $\left(v^{M_{k}}, V^{M_{k}}\right) \in H_{0, \partial D}^{1}(\Omega \backslash \bar{D}) \oplus T^{M_{k}}$ and $v \in H_{0, \partial D}^{1}(\Omega \backslash \bar{D})$ be the solutions corresponding to the operator current pairs $\left(\left(L^{M_{k}}\right)^{\prime}, P^{M_{k}} g\right)$ and $\left(\tilde{L}^{\prime}, g\right)$, respectively; i.e., by (3.8) and (3.37), $\left(L^{M_{k}}\right)^{\prime} P^{M_{k}} g=\left.v^{M_{k}}\right|_{\partial D}$ and $\tilde{L}^{\prime} g=\left.v\right|_{\partial D}$. Since $\phi^{M_{k}} \in$ $H_{0}^{-1 / 2}(\partial D)$, by a slight variation of Lemma 2.7 and Theorem 2.4, we have

$$
\begin{aligned}
\left|\left\langle\phi^{M_{k}},\left(\left(L^{M_{k}}\right)^{\prime} P^{M_{k}}-\tilde{L}^{\prime}\right) g\right\rangle_{L^{2}(\partial D)}\right| \leq & \left\|\phi^{M_{k}}\right\|_{H^{-1 / 2}(\partial D)}\left\|v^{M_{k}}-v\right\|_{H^{1 / 2}(\partial D) / \mathbb{R}} \\
\leq & C\left\|v^{M_{k}}-v\right\|_{H^{1}(\Omega \backslash \bar{D}) / \mathbb{R}} \\
\leq & C\left\{\inf _{V \in T^{M_{k}}}\|(v-z g)-V\|_{L^{2}\left(\Gamma_{M_{k}}\right)}\right. \\
& \left.+\frac{1}{\left|\Gamma_{M_{k}}\right|^{1 / 2}}\|g\|_{\tilde{H}^{-1 / 2}\left(\partial \Omega \backslash \bar{\Gamma}_{M_{k}}\right)}\right\} \rightarrow 0,
\end{aligned}
$$


when $k$ goes to infinity by Lemma 3.11 and (3.33) since $\left.v\right|_{\partial \Omega}-z g \in C^{\infty}(\partial \Omega)$ due to the assumptions on $z$ and $g$, and the regularity theory of elliptic partial differential equations [10]. Finally, due to the weak convergence of $\left\{\phi^{M_{k}}\right\}$, the second term on the right-hand side of (3.43) satisfies

$$
\left\langle\phi^{M_{k}}, \tilde{L}^{\prime} g\right\rangle_{L^{2}(\partial D)} \rightarrow\left\langle\phi^{\prime}, \tilde{L}^{\prime} g\right\rangle_{L^{2}(\partial D)}=\left\langle\tilde{L} \phi^{\prime}, g\right\rangle_{L^{2}(\partial \Omega)},
$$

when $k$ goes to infinity.

Putting the above estimates together, we have established that

$$
\left\langle L^{M_{k}} \phi^{M_{k}}, g\right\rangle_{L^{2}(\partial \Omega)} \rightarrow\left\langle\tilde{L} \phi^{\prime}, g\right\rangle_{L^{2}(\partial \Omega)}=\left\langle\Phi_{y}, g\right\rangle_{L^{2}(\partial \Omega)} \quad \text { when } k \rightarrow \infty,
$$

for all $g \in C^{\infty}(\partial \Omega) \cap L_{0}^{2}(\partial \Omega)$, by the first part of the proof. This means that $\tilde{L} \phi^{\prime}=$ $\left.\Phi_{y}\right|_{\partial \Omega}$ almost everywhere on $\partial \Omega$, which is the contradiction we were looking for.

Then it is the turn of $y \in D$.

Lemma 3.13. Assume that $y \in D$ and let $\left\{\alpha_{M}\right\} \subset \mathbb{R}_{+}$be such that the sequence

$$
\left\{\frac{\inf _{V \in T^{M}}\left\|\Phi_{y}-V\right\|_{L^{2}(\partial \Omega)}^{2}}{\alpha_{M}}\right\}
$$

is bounded. Then the sequence of the minimizers $\left\{\phi^{M}\right\} \subset H_{0}^{-1 / 2}(\partial D)$ for (3.38) also is bounded.

Proof. To begin with, note that $\left.{\frac{\partial \Phi_{y}}{\partial \nu}}^{+}\right|_{\partial D} \in H_{0}^{-1 / 2}(\partial D)$ due to the divergence theorem. Since $L^{M}{\frac{\partial \Phi_{y}}{\partial \nu}}^{+} \in L_{0}^{2}\left(\Gamma_{M}\right)$ and, clearly, $\tilde{L}{\frac{\partial \Phi_{y}}{\partial \nu}}^{+}=\left.\Phi_{y}\right|_{\partial \Omega} \in H_{0}^{1 / 2}(\partial \Omega)$, as in the proof of Lemma 3.12, we have the estimate

$$
\left\|L^{M} \frac{\partial \Phi_{y}^{+}}{\partial \nu}-\Phi_{y}\right\|_{L^{2}(\partial \Omega)}^{2}=\left\|\left(L^{M}-\tilde{L}\right) \frac{\partial \Phi_{y}}{\partial \nu}\right\|_{L^{2}(\partial \Omega)}^{2} \leq \frac{C}{\left|\Gamma_{M}\right|} \inf _{V \in T^{M}}\left\|\Phi_{y}-V\right\|_{L^{2}(\partial \Omega)}^{2} .
$$

Thus, due to the minimizing property of the sequence $\left\{\phi^{M}\right\} \subset H_{0}^{-1 / 2}(\partial D)$, for every $M \in \mathbb{N}$, we have

$$
\begin{aligned}
\left\|L^{M} \phi^{M}-\Phi_{y}\right\|_{L^{2}(\partial \Omega)}^{2} & +\alpha_{M}\left\|\phi^{M}\right\|_{H^{-1 / 2}(\partial D)}^{2} \\
& \leq \frac{C}{\left|\Gamma_{M}\right|} \inf _{V \in T^{M}}\left\|\Phi_{y}-V\right\|_{L^{2}(\partial \Omega)}^{2}+\alpha_{M}\left\|\frac{\partial \Phi_{y}}{\partial \nu}\right\|_{H^{-1 / 2}(\partial D)}^{2}
\end{aligned}
$$

Forgetting the first term on the left-hand side of (3.45) and dividing by $\alpha_{M}$, we get

$$
\left\|\phi^{M}\right\|_{H^{-1 / 2}(\partial D)}^{2} \leq C \frac{\inf _{V \in T^{M}}\left\|\Phi_{y}-V\right\|_{L^{2}(\partial \Omega)}^{2}}{\alpha_{M}\left|\Gamma_{M}\right|}+\left\|\frac{\partial \Phi_{y}}{\partial \nu}\right\|_{H^{-1 / 2}(\partial D)}^{2},
$$

for every $M \in \mathbb{N}$. Together with assumptions (3.44) and (3.33), this proves the claim.

If the operator sequence $\left\{L^{M}\right\}$ is known, Lemmas 3.12 and 3.13 give us the means to find the inclusion $D$. However, to know $\left\{L^{M}\right\}$ is to know the shape of the boundary $\partial D$. Luckily, the operators $L^{M}$ and $\left|R_{\sigma}^{M}-R_{1}^{M}\right|^{1 / 2}$ are closely related, and so Lemmas 3.12 and 3.13 give us the weaponry to write out the proof for Theorem 3.10.

Proof of Theorem 3.10. Let us define a new sequence $\left\{\tilde{\phi}^{M}\right\} \subset H_{0}^{-1 / 2}(\partial D), \tilde{\phi}^{M} \in$ $\mathcal{R}\left(F^{M}\left(L^{M}\right)^{\prime}\right)$, by

$$
\tilde{\phi}^{M}=\left(L^{M}\right)^{-1}\left|R_{\sigma}^{M}-R_{1}^{M}\right|^{1 / 2} I_{M}, \quad 1 \leq M<\infty,
$$


where $\left(L^{M}\right)^{-1}$ is given by (3.32). We get a simple relation between the norms of $I^{M}$ and $\tilde{\phi}^{M}$ :

$$
\begin{aligned}
\left\|I^{M}\right\|_{L^{2}(\partial \Omega)}^{2} & =\left\langle\left|R_{\sigma}^{M}-R_{\mathbf{1}}^{M}\right|^{-1 / 2} L^{M} \tilde{\phi}^{M},\left|R_{\sigma}^{M}-R_{\mathbf{1}}^{M}\right|^{-1 / 2} L^{M} \tilde{\phi}^{M}\right\rangle_{L^{2}(\partial \Omega)} \\
& =\left\langle\tilde{\phi}^{M},\left(L^{M}\right)^{\prime}\left|R_{\sigma}^{M}-R_{\mathbf{1}}^{M}\right|^{-1} L^{M} \tilde{\phi}^{M}\right\rangle_{L^{2}(\partial D)} \\
& =\left\langle\tilde{\phi}^{M},\left(L^{M}\right)^{\prime}\left(\left(L^{M}\right)^{\prime}\right)^{-1}\left|F^{M}\right|^{-1}\left(L^{M}\right)^{-1} L^{M} \tilde{\phi}^{M}\right\rangle_{L^{2}(\partial D)} \\
& =\left\langle\tilde{\phi}^{M},\left|F^{M}\right|^{-1} \tilde{\phi}^{M}\right\rangle_{L^{2}(\partial D)}=\left\|\left(F^{M}\right)^{-1 / 2} \tilde{\phi}^{M}\right\|_{L^{2}(\partial D)}^{2}
\end{aligned}
$$

In consequence, since the sequence $\left\{I^{M}\right\}$ minimizes the functionals (3.35), the sequence $\left\{\tilde{\phi}^{M}\right\}$ minimizes the functionals

$$
\left\|L^{M} \phi-\Phi_{y}\right\|_{L^{2}(\partial \Omega)}^{2}+\alpha_{M}\left\|\left(F^{M}\right)^{-1 / 2} \phi\right\|_{L^{2}(\partial D)}^{2}, \quad 1 \leq M<\infty,
$$

within the subspaces $\mathcal{R}\left(F^{M}\left(L^{M}\right)^{\prime}\right)$, respectively. Indeed, if $\hat{\phi}^{M} \in \mathcal{R}\left(F^{M}\left(L^{M}\right)^{\prime}\right)$ gave a smaller value for functional (3.46), then one easily sees that $\left|R_{\sigma}^{M}-R_{1}^{M}\right|^{-1 / 2} L^{M} \hat{\phi}^{M} \in$ $T_{0}^{M}$ would give a smaller value than $I^{M}$ for functional (3.35), which is a contradiction.

We define yet a new sequence by $\left\{\phi^{M}\right\}=\left\{Q^{M} \tilde{\phi}^{M}\right\}$, where $Q^{M}: \mathcal{R}\left(F^{M}\left(L^{M}\right)^{\prime}\right) \rightarrow$ $\mathcal{N}\left(L^{M}\right)^{\perp} \subset H_{0}^{-1 / 2}(\partial D)$ is defined by (3.29). Here and in the rest of this proof the orthogonal complement $\mathcal{N}\left(L^{M}\right)^{\perp}$ is taken with respect to the $H^{-1 / 2}$ inner product. By similar reasoning as above, one sees that this new sequence minimizes the functionals

$$
\left\|L^{M} \phi-\Phi_{y}\right\|_{L^{2}(\partial \Omega)}^{2}+\alpha_{M}\left\|\left(F^{M}\right)^{-1 / 2}\left(Q^{M}\right)^{-1} \phi\right\|_{L^{2}(\partial D)}^{2}, \quad 1 \leq M<\infty,
$$

over the subspaces $\mathcal{N}\left(L^{M}\right)^{\perp}$, respectively. Now, Lemma 3.8 and Corollary 3.9 tell us that there exists a sequence of functionals $\left\{C_{M}\right\}, C_{M}: \mathcal{N}\left(L^{M}\right)^{\perp} \rightarrow \mathbb{R}$, such that

$$
\left\|\left(F^{M}\right)^{-1 / 2}\left(Q^{M}\right)^{-1} \phi\right\|_{L^{2}(\partial D)}=C_{M}(\phi)\|\phi\|_{H^{-1 / 2}(\partial D)}, \quad c \leq C_{M} \leq C,
$$

for all $M \in \mathbb{N}$ and $\phi \in \mathcal{N}\left(L^{M}\right)^{\perp}$, where $c$ and $C$ are positive constants independent of $M$. Thus, the sequence $\left\{\phi^{M}\right\}$ also minimizes the functionals

$$
\left\|L^{M} \phi-\Phi_{y}\right\|_{L^{2}(\partial \Omega)}^{2}+\alpha_{M} C_{M}^{2}(\phi)\|\phi\|_{H^{-1 / 2}(\partial D)}^{2}, \quad 1 \leq M<\infty,
$$

over the subspaces $\mathcal{N}\left(L^{M}\right)^{\perp} \subset H_{0}^{-1 / 2}(\partial D)$, respectively. In particular, if we define $C_{M}(\phi)=C$ for $\phi \in H_{0}^{-1 / 2}(\partial D) \backslash \mathcal{N}\left(L^{M}\right)^{\perp}$, the sequence $\left\{\phi^{M}\right\}$ minimizes functionals (3.47) over the whole space $H_{0}^{-1 / 2}(\partial D)$. It is an easy consequence of the upper and lower bounds for $\left\{C_{M}\right\}$ that $\left\{\phi^{M}\right\}$ is bounded in $H_{0}^{-1 / 2}(\partial D)$ if and only if $y \in D$.

Let $\left\{\phi_{c}^{M}\right\},\left\{\phi_{C}^{M}\right\} \subset H_{0}^{-1 / 2}(\partial D)$ be the minimizing sequences for the functionals

$$
\left\|L^{M} \phi-\Phi_{y}\right\|_{L^{2}(\partial \Omega)}^{2}+\frac{1}{2} \alpha_{M} c^{2}\|\phi\|_{H^{-1 / 2}(\partial D)}^{2}, \quad 1 \leq M<\infty,
$$

and

$$
\left\|L^{M} \phi-\Phi_{y}\right\|_{L^{2}(\partial \Omega)}^{2}+2 \alpha_{M} C^{2}\|\phi\|_{H^{-1 / 2}(\partial D)}^{2}, \quad 1 \leq M<\infty,
$$


respectively. It follows from Lemmas 3.12 and 3.13 that each of these sequences is bounded if and only if $y \in D$. Let us shorten our strenuous notations by $\Psi_{M}(\phi)=$ $\left\|L^{M} \phi-\Phi_{y}\right\|_{L^{2}(\partial \Omega)}$ and note that due to the minimizing properties of the sequences $\left\{\phi^{M}\right\}$ and $\left\{\phi_{c}^{M}\right\}$, for every $M \in \mathbb{N}$, we have

$\Psi_{M}^{2}\left(\phi^{M}\right)+\alpha_{M} C_{M}^{2}\left(\phi^{M}\right)\left\|\phi^{M}\right\|_{H^{-1 / 2}(\partial D)}^{2} \leq \Psi_{M}^{2}\left(\phi_{c}^{M}\right)+\alpha_{M} C_{M}^{2}\left(\phi_{c}^{M}\right)\left\|\phi_{c}^{M}\right\|_{H^{-1 / 2}(\partial D)}^{2}$,

and

$$
\Psi_{M}^{2}\left(\phi^{M}\right)+\frac{1}{2} \alpha_{M} c^{2}\left\|\phi^{M}\right\|_{H^{-1 / 2}(\partial D)}^{2} \geq \Psi_{M}^{2}\left(\phi_{c}^{M}\right)+\frac{1}{2} \alpha_{M} c^{2}\left\|\phi_{c}^{M}\right\|_{H^{-1 / 2}(\partial D)}^{2} .
$$

By subtracting the second of these inequalities from the first one and arranging terms, we get

$$
\left\|\phi^{M}\right\|_{H^{-1 / 2}(\partial D)}^{2} \leq \frac{C_{M}^{2}\left(\phi_{c}^{M}\right)-\frac{1}{2} c^{2}}{C_{M}^{2}\left(\phi^{M}\right)-\frac{1}{2} c^{2}}\left\|\phi_{c}^{M}\right\|_{H^{-1 / 2}(\partial D)}^{2} \leq \frac{2 C^{2}-c^{2}}{c^{2}}\left\|\phi_{c}^{M}\right\|_{H^{-1 / 2}(\partial D)}^{2} .
$$

On the other hand, by similar means we deduce that

$$
\left\|\phi^{M}\right\|_{H^{-1 / 2}(\partial D)}^{2} \geq \frac{2 C^{2}-C_{M}^{2}\left(\phi_{C}^{M}\right)}{2 C^{2}-C_{M}^{2}\left(\phi^{M}\right)}\left\|\phi_{C}^{M}\right\|_{H^{-1 / 2}(\partial D)}^{2} \geq \frac{C^{2}}{2 C^{2}-c^{2}}\left\|\phi_{C}^{M}\right\|_{H^{-1 / 2}(\partial D)}^{2} .
$$

From the above estimates it follows that $\left\{\phi^{M}\right\} \subset H_{0}^{-1 / 2}(\partial D)$ is bounded if and only if $y \in D$.

Finally, walking the above path of reasoning backwards, one sees that

$$
\left\|I^{M}\right\|_{L^{2}(\partial \Omega)}=\left\|\left(F^{M}\right)^{-1 / 2}\left(Q^{M}\right)^{-1} \phi^{M}\right\|_{L^{2}(\partial D)},
$$

and so the claim follows from the uniform boundedness of the operator sequences $\left\{\left(F^{M}\right)^{1 / 2}\right\},\left\{\left(F^{M}\right)^{-1 / 2}\right\}$, and $\left\{Q^{M}\right\},\left\{\left(Q^{M}\right)^{-1}\right\}$ given in Lemma 3.8 and Corollary 3.9 , respectively.

We end this section, and at the same time the whole work, by noting that one could easily modify Theorem 3.10 for the case of multiple inclusions by using the means described in [3].

\section{REFERENCES}

[1] L. Borcea, Electrical impedance tomography, Inverse Problems, 18 (2002), pp. R99-R136.

[2] D. Braess, Finite Elements: Theory, Fast Solvers, and Applications in Solid Mechanics, 2nd ed., Cambridge University Press, Cambridge, UK, 2001.

[3] M. BRÜHL, Explicit characterization of inclusions in electrical impedance tomography, SIAM J. Math. Anal., 32 (2001), pp. 1327-1341.

[4] F. Cakoni and D. Colton, The linear sampling method for cracks, Inverse Problems, 19 (2003), pp. 279-295.

[5] R. Dautray and J-L. Lions, Mathematical Analysis and Numerical Methods for Science and Technology, Vol. 2, Springer-Verlag, Berlin, 1988.

[6] V. Hutson And J. S. Pym, Applications of Functional Analysis and Operator Theory, Academic Press, London, 1980.

[7] A. Kirsch, Characterization of the shape of the scattering obstacle using the spectral data of the far field operator, Inverse Problems, 14 (1998), pp. 1489-1512.

[8] O. A. Ladyzhenskaya, The Boundary Value Problems of Mathematical Physics, SpringerVerlag, New York, 1985. 
[9] E. Somersalo, M. Cheney, and D. Isaacson, Existence and uniqueness for electrode models for electric current computed tomography, SIAM J. Appl. Math., 52 (1992), pp. 1023-1040.

10] M. E. TAYlor, Partial Differential Equations I, Springer-Verlag, New York, 1996.

[11] M. Vauhkonen, D. Vadász, P. A. Karjalainen, E. Somersalo, and J. P. Kaipio, Tikhonov regularization and prior information in electrical impedance tomography, IEEE Trans. Med. Imaging, 17 (1998), pp. 285-293.

[12] P. Vauhkonen, M. Vauhkonen, T. Savolainen, and J. Kaipio, Three-dimensional electrical impedance tomography based on the complete electrode model, IEEE Trans. Biomed. Eng., 46 (1999), pp. 1150-1160.

[13] K. YosidA, Functional Analysis, 6th ed., Springer-Verlag, New York, 1980. 